\title{
An Extended Car-Following Model considering the Driver's Desire for Smooth Driving and Self-Stabilizing Control with Velocity Uncertainty
}

\author{
Shihao Li, ${ }^{1,2,3}$ Ting Wang, ${ }^{1,2,3}$ Rongjun Cheng $\mathbb{D}^{1,2,3}$ and Hongxia Ge $\mathbb{D}^{1,2,3}$ \\ ${ }^{1}$ Faculty of Maritime and Transportation, Ningbo University, Ningbo 315211, China \\ ${ }^{2}$ Jiangsu Province Collaborative Innovation Center for Modern Urban Traffic Technologies, Nanjing 210096, China \\ ${ }^{3}$ National Traffic Management Engineering and Technology Research Centre Ningbo University Sub-Centre, \\ Ningbo 315211, China
}

Correspondence should be addressed to Rongjun Cheng; chengrongjun76@126.com

Received 9 July 2020; Accepted 14 July 2020; Published 29 August 2020

Guest Editor: Miaojuan Peng

Copyright (c) 2020 Shihao Li et al. This is an open access article distributed under the Creative Commons Attribution License, which permits unrestricted use, distribution, and reproduction in any medium, provided the original work is properly cited.

In this paper, an extended car-following model with consideration of the driver's desire for smooth driving and the self-stabilizing control in historical velocity data is constructed. Moreover, for better reflecting the reality, we also integrate the velocity uncertainty into the new model to analyze the internal characteristics of traffic flow in situation where the historical velocity data are uncertain. Then, the model's linear stability condition is inferred by utilizing linear stability analysis, and the modified Kortewegde Vries ( $\mathrm{mKdV}$ ) equation is also obtained to depict the evolution properties of traffic congestion. According to the theoretical analysis, we observe that the degree of traffic congestion is alleviated when the control signal is considered, and the historical time gap and the velocity uncertainty also play a role in affecting the stability of traffic flow. Finally, some numerical simulation experiments are implemented and the experiments' results demonstrate that the control signals including the self-stabilizing control, the driver's desire for smooth driving, the historical time gap, and the velocity uncertainty are of avail to improve the traffic jam, which are consistent with the theoretical analytical results.

\section{Introduction}

Along with the improvement of modern transportation system, the complexity of transportation system is becoming larger and larger. Thus, there are more concerns about the fact that the transportation system's growing complexity will undoubtedly increase the burden of people's travel. A large number of researchers have been involved in the research of revealing the dynamic properties of traffic flow over the years [1-5]. In the past few decades, lots of models based on the actual traffic conditions have been built to study traffic flow. Normally, traffic flow models can be categorized into microscopic models [1, 6-12], lattice models [3, 4, 13-22], and macroscopic hydrodynamic models [23-32].

The car-following model theory aims at analyzing the motion properties of a single vehicle and establishes the relationship between current vehicle and preceding vehicle on the road without the case of overtaking. Besides, the carfollowing model can describe many evolution processes of traffic flow and many traffic factors can be integrated into the car-following model to simulate real traffic situations [33-36]. It has now been almost seventy years since the first paper about car-following model was published, which was constructed by Pipes [37] in 1953. However, the acceleration process of single vehicle cannot be described by the model correctly. Later in 1961, Newell [38] suggested a novel carfollowing model, which established the expression of velocity instead of acceleration. But the acceleration of vehicle will become infinite when the traffic light changes to green from red in intersection. For solving the defects of previous models, Bando et al. [39] proposed the classical optimal velocity model (OVM). In OVM, the delay time of vehicle is 
not considered. Moreover, the detailed vehicle characters and realistic driver behavior are included in OVM, many traffic phenomena observed in real traffic can be simulated in terms of OVM. Since then, on account of the OVM, a legion of researchers proposed new models integrating different traffic factors to study traffic flow. In 1998, Helbing and Tilch [40] identified the OVM by utilizing the measured information and the simulation results showed that the OVM can generate the unrealistic deceleration and acceleration rates. Hence, Helbing et al. suggested the general force model (GFM). In 2001, based on the GFM, Jiang et al. [41] constructed the full velocity difference model (FVDM) with consideration of the effect of positive velocity difference on vehicle dynamics.

In real traffic environment, on the one hand, drivers wish to keep vehicle's velocity consistent with traffic flow's equilibrium velocity for reducing the frequency of adjusting speed. With that in mind, Wang et al. [42] proposed the extended car-following model considering the driver's desire for smooth driving in the difference between current and historical velocity. In their studies, some simulation experiments were implemented to verify the impact of the driver's desire for smooth driving, and the results corroborated that the factor plays a positive role in stabilizing traffic flow. On the other hand, the desire for driving smoothly is just one of drivers' psychological characteristics, because the drivers may also wish to keep vehicle's speed consistent with the velocity of previous moment for reducing energy consumption. Based on this, Li et al. [43] constructed the novel car-following model taking the self-stabilizing control effect in the difference of historical velocity data into account. From the investigation by using analytical and numerical methods, researchers found that the traffic flow will be more stable by means of the self-stabilizing control, just like the cooperatively driving control. According to the analysis above, we can conclude that the driver's desire for smooth driving and the self-stabilizing effect represent the drivers' two psychological characteristics; different drivers will choose different driving strategies. Meanwhile, we can realize that the historical velocity data can be introduced into traffic flow models to study traffic flow's properties.

Recently, many researchers believe that the operation of intelligent transportation system will be affected by numerous uncertain factories, such as network fluctuation, fault of equipment, driver's personality, and traffic interruption. Hence, the historical traffic data including historical velocity is not usually accurate when uncertain factories happen. With that in mind, Zhang et al. [44] put forward an enhanced FVDM incorporating the velocity uncertainty in cooperation driving system and found that velocity uncertainty of preceding vehicle has a significant influence on traffic flow. Nevertheless, relatively little attention has been drawn toward velocity uncertainty of current vehicle. In reality, the driver will not always pay attention to the velocity of current vehicle; there will be a difference between the velocity perceived by driver and the actual velocity of the current vehicle. The velocity uncertainty of this study indicates the difference between real velocity and velocity perceived by drivers in historical time.

In conclusion, for better mirroring the reality and revealing the evolution characteristics of traffic flow when these traffic conditions are taken into account, this paper constructed an enhanced car-following model incorporating the driver's desire for smooth driving and the self-stabilizing control with velocity uncertainty in Section 2 . The remainder of the paper is arranged as follows. In Section 3, linear stability analysis of enhanced model is derived. The $\mathrm{mKdV}$ equation is inferred to display the evolution properties of traffic congestion in Section 4. After that, some simulation experiments are conducted to verify the correctness of numerical analysis and to discuss the influences of proposed factors on traffic flow in Section 5. The final section gives some conclusions.

\section{The Improved Model}

Bando et al. established the well-known OVM, and the dynamic formula of OVM can be shown as

$$
\frac{\mathrm{d}^{2} x_{n}(t)}{\mathrm{d} t^{2}}=\alpha\left[V^{o p}\left(\Delta x_{n}(t)\right)-v_{n}(t)\right]
$$

where $\alpha$ represents the sensitivity coefficient of drivers; $\Delta x_{n}(t)=x_{n+1}(t)-x_{n}(t)$ stands for distance between vehicle $n$ and vehicle $n+1 ; v_{n}(t)$ represents velocity of vehicle $n$; the desired optimal velocity of vehicle $n$ is as follows:

$$
V^{o p}\left(\Delta x_{n}(t)\right)=\frac{v_{\max }}{2}\left[\tanh \left(\Delta x_{n}(t)-h_{c}\right)+\tanh \left(h_{c}\right)\right]
$$

where $v_{\max }$ and $h_{c}$ represent the maximum speed and the safety space headway distance.

Since the historical velocity information of current vehicle is uncertain, we introduce the effects of the driver's desire for smooth driving and self-stabilizing control with velocity uncertainty on the OVM, and the extended model can be expressed as

$$
\frac{\mathrm{d}^{2} x_{n}(t)}{\mathrm{d} t^{2}}=\alpha\left[V^{o p}\left(y_{n}(t)\right)-v_{n}(t)\right]+\lambda_{1}\left[V(h)-(1+p) v_{n}(t-\tau)\right]+\lambda_{2}\left[v_{n}(t)-(1+p) v_{n}(t-\tau)\right]
$$

where $y_{n}(t)=\Delta x_{n}(t) ; h$ represents the average space headway distance; $\tau$ is the time gap between the historical time and the current time; $V(h)-v_{n}(t-\tau)$ denotes the driver's desire for smooth driving; $v_{n}(t)-v_{n}(t-\tau)$ denotes the self-stabilizing control effect in the difference between the current and history velocity; $\lambda_{1}$ and $\lambda_{2}$ mean the reaction coefficients of two introduced factors, respectively; $p$ denotes the reaction coefficient reflecting the uncertainty of vehicle's 


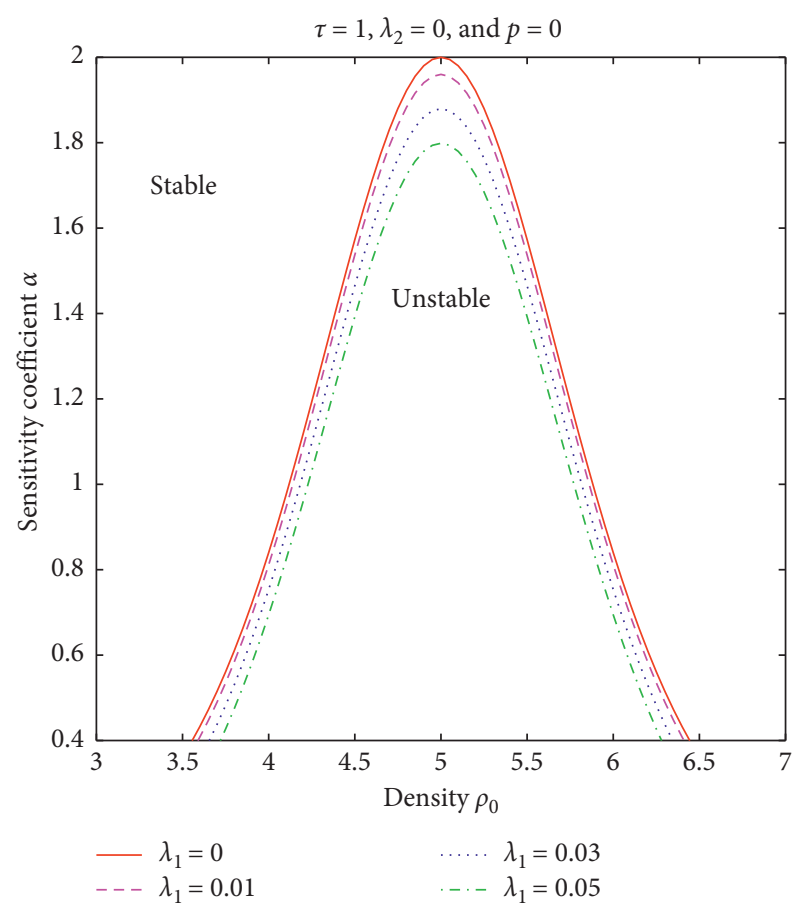

Figure 1: The neutral stability lines for different $\lambda_{1}$ with $\lambda_{2}=0, p=0$, and $\tau=1$.

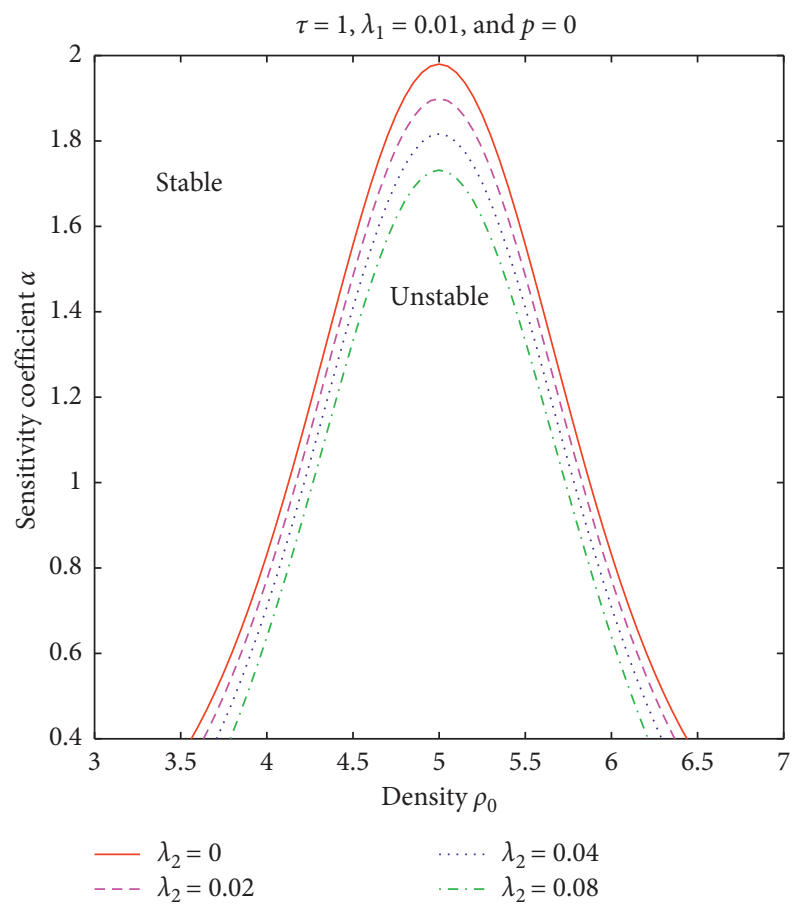

Figure 2: The neutral stability lines for different $\lambda_{2}$ with $\lambda_{1}=0.01, p=0$, and $\tau=1$.

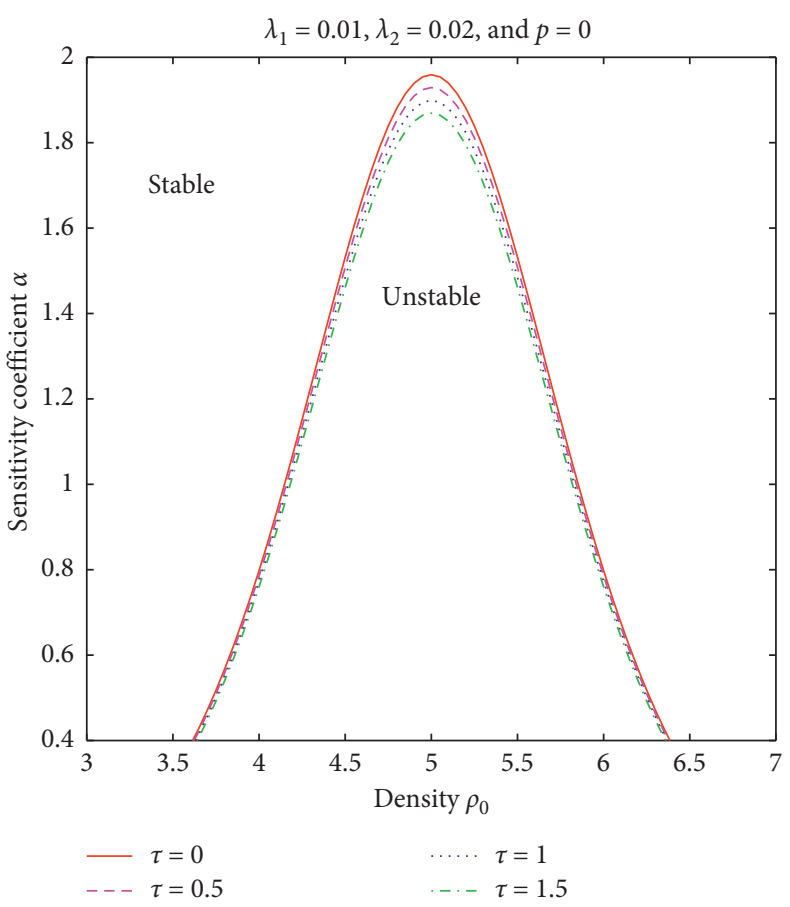

Figure 3: The neutral stability lines for different $\tau$ with $\lambda_{1}=0.01, \lambda_{2}=0.02$, and $p=0$.

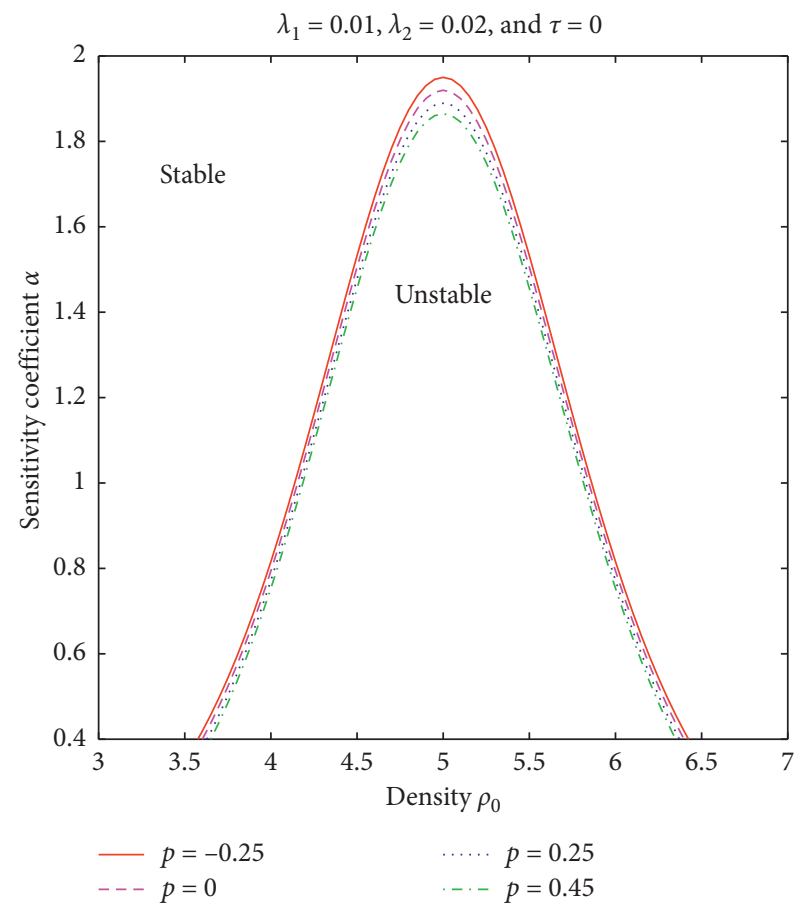

FIgURE 4: The neutral stability lines for different $p$ with $\lambda_{1}=0.01, \lambda_{2}=0.02$, and $\tau=1$. 


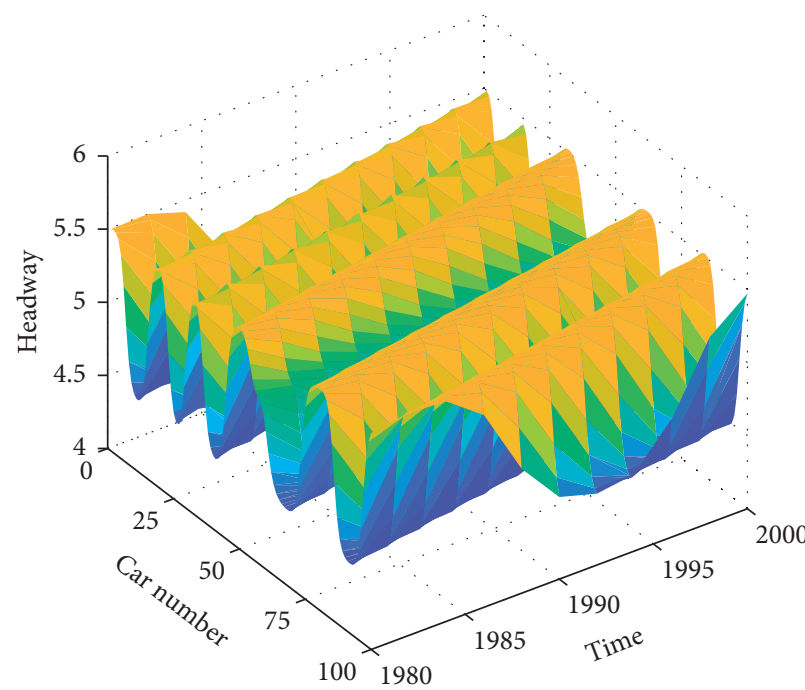

(a)

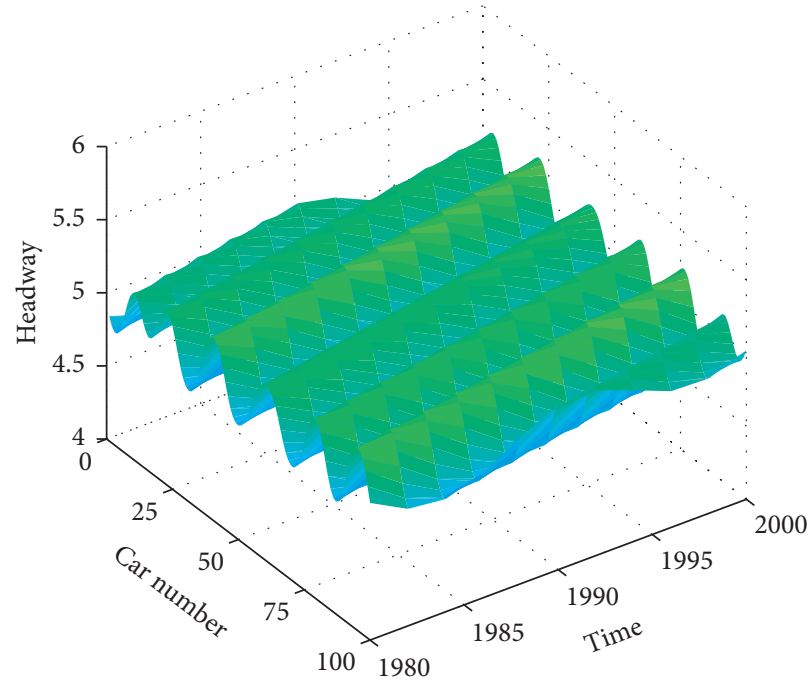

(c)

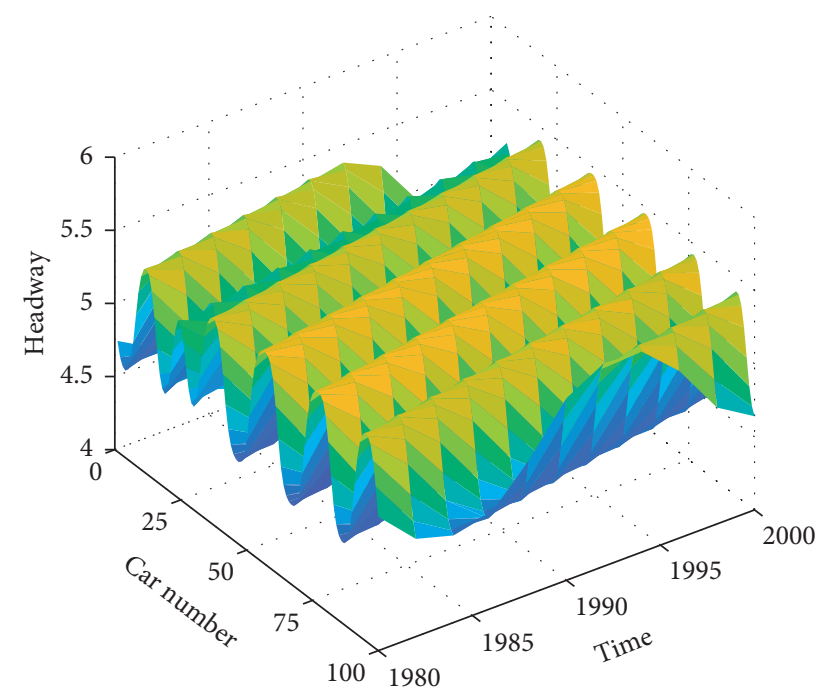

(b)

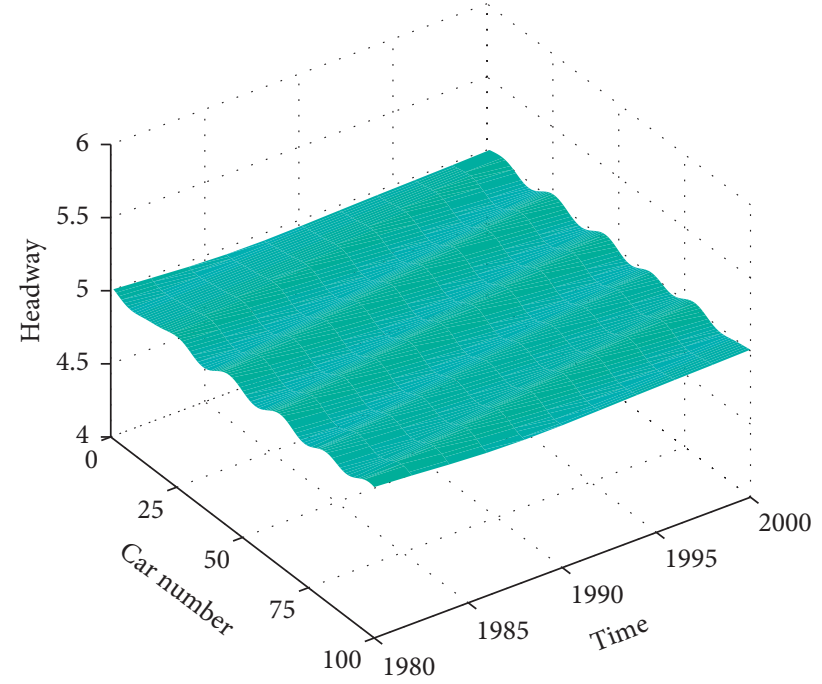

(d)

FIGURE 5: The profiles of headway for different $\lambda_{1}$ under $\lambda_{2}=0, p=0$, and $\tau=1$. (a) $\lambda_{1}=0$ and $\lambda_{2}=0$, (b) $\lambda_{1}=0.01$ and $\lambda_{2}=0$, (c) $\lambda_{1}=0.02$ and $\lambda_{2}=0$, and (d) $\lambda_{1}=0.03$ and $\lambda_{2}=0$.

velocity; $-1<p<0$ stands for the vehicle's real velocity which is larger than the historical velocity perceived by drivers, impelling that the drivers overestimate the driving condition and choose to increase the acceleration of vehicle, which will likely cause the traffic accident and traffic jam; $p=0$ means that the historical velocity perceived by drivers equals the vehicle's real velocity; $0<p<1$ represents the historical velocity perceived by drivers which is larger than the vehicle's real velocity, spurring that the drivers underestimate the driving condition and make a deceleration decision, which will probably cause the inessential delay and traffic congestion.

From (3), we can get the space headway equation of novel model which is written as

$$
\frac{\mathrm{d}^{2} \Delta x_{n}(t)}{\mathrm{d} t^{2}}=\alpha\left[V\left(\Delta x_{n+1}(t)\right)-V\left(\Delta x_{n}(t)\right)\right]+\left(\lambda_{2}-\alpha\right) \frac{\mathrm{d} \Delta x_{n}(t)}{\mathrm{d} t}-\left(\lambda_{1}+\lambda_{2}\right)(1+p) \frac{\mathrm{d} \Delta x_{n}(t-\tau)}{\mathrm{d} t}
$$

\section{Linear Stability Analysis}

The method of linear stability analysis will be utilized to obtain the stability condition of enhanced model in this section. First of all, let $N$ represent the number of all vehicles and $L$ denote the road's length. Traffic flow's steady state will be defined as that all vehicles possess the same headway and velocity. Then the uniform steady state of extended model is 


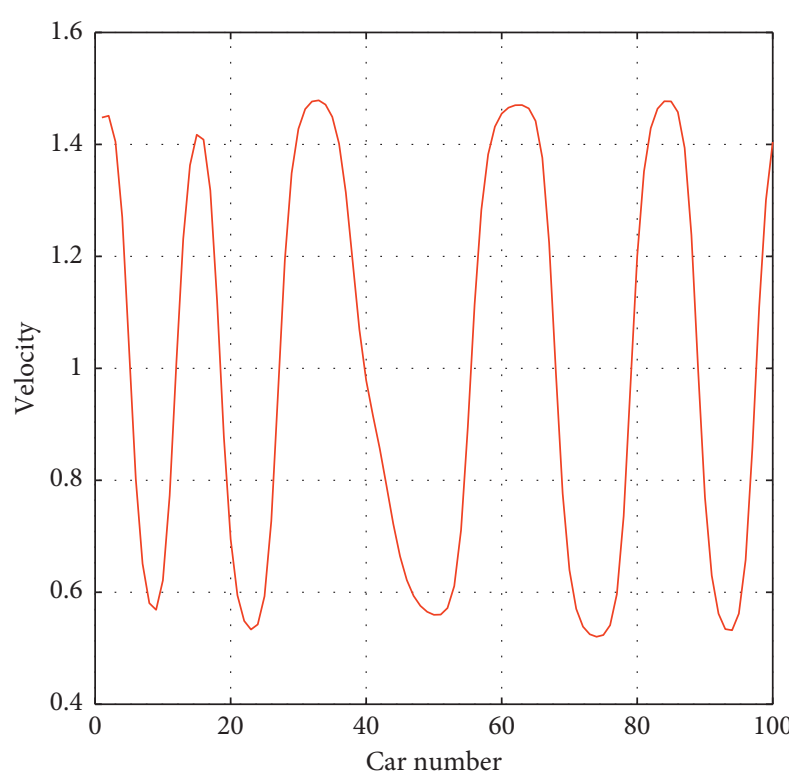

(a)

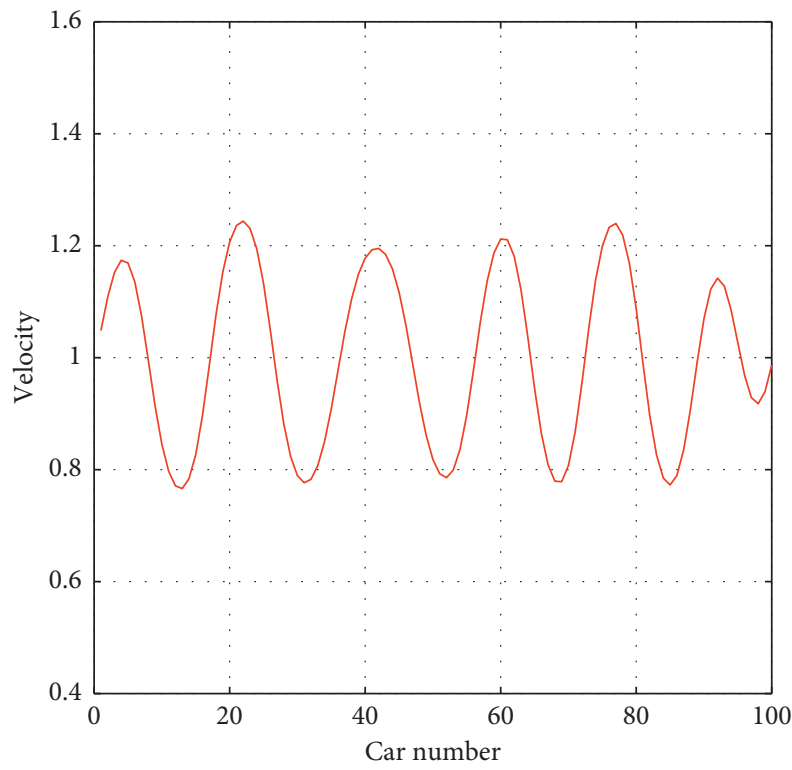

(c)

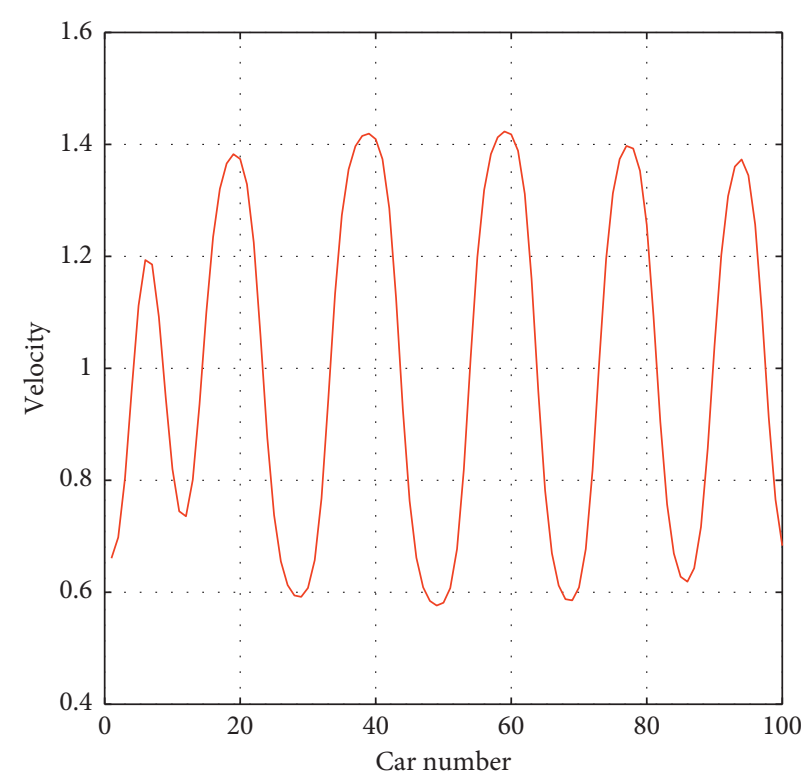

(b)

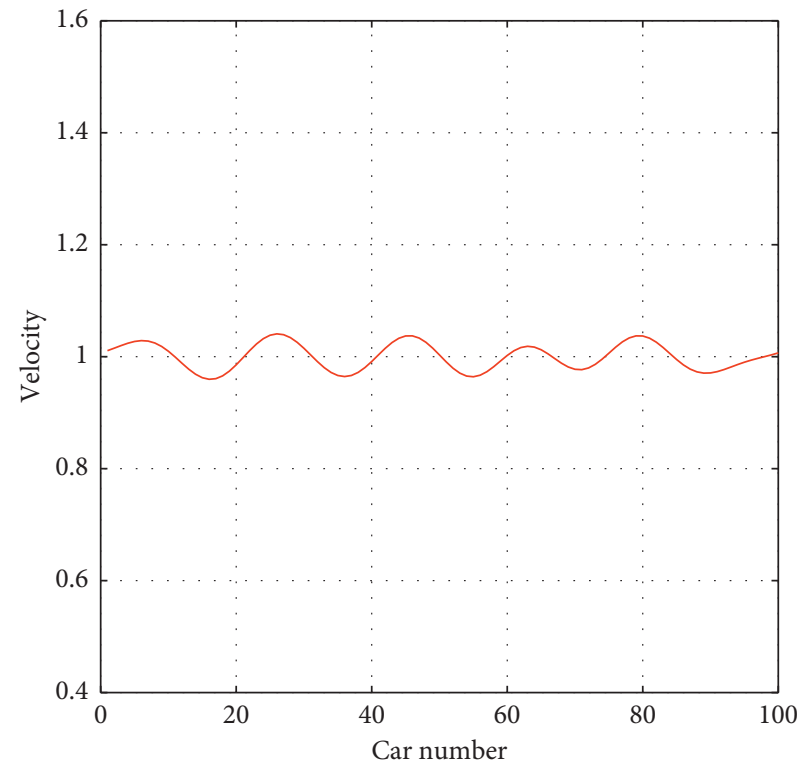

(d)

Figure 6: The profiles of velocity for different $\lambda_{1}$ under $\lambda_{2}=0, p=0$, and $\tau=1$. (a) $\lambda_{1}=0$ and $\lambda_{2}=0$, (b) $\lambda_{1}=0.01$ and $\lambda_{2}=0$, (c) $\lambda_{1}=0.02$ and $\lambda_{2}=0$, and (d) $\lambda_{1}=0.03$ and $\lambda_{2}=0$.

$$
\begin{aligned}
\Delta x_{n}^{*}(t) & =h, \\
v_{n}^{*}(t) & =V(h),
\end{aligned}
$$

where $h$ equals $(L / N)$.

Supposing that $\varepsilon_{n}(t)$ represents a small perturbation, we impose it into the steady state to gain the linear stability condition. Then the new state of traffic flow can be represented as

$$
\Delta x_{n}(t)=h+\varepsilon_{n}(t)
$$

Substituting (6) into (4), we can get the new equation which can be expressed as

$$
\begin{aligned}
z^{2}=\alpha & {\left[V^{\prime}(h)\left(\varepsilon_{n+1}(t)-\varepsilon_{n}(t)\right)\right]-\left(\lambda_{1}+\lambda_{2}\right)(1+p) } \\
\cdot & \frac{\mathrm{d} \varepsilon_{n}(t-\tau)}{\mathrm{d} t}+\left(\lambda_{2}-\alpha\right) \frac{\mathrm{d} \varepsilon_{n}(t)}{\mathrm{d} t} .
\end{aligned}
$$

Transforming $\varepsilon_{n}(t)$ into Fourier form of $\varepsilon_{n}(t)=A \quad \exp (i k n+z t)$, then (7) will be translated to

$$
z^{2}=\alpha V^{\prime}(h)\left(e^{i k}-1\right)+\left(\lambda_{2}-\alpha\right) z-\left(\lambda_{1}+\lambda_{2}\right)(1+p) z e^{-z \tau} .
$$

Further expanding $z$ and $e^{i k}$ with the form of $z \approx z_{1}(i k)+z_{2}(i k)^{2}$ and $e^{i k} \approx 1+(i k)+\left(i k^{2} / 2\right)$ in (8), we 


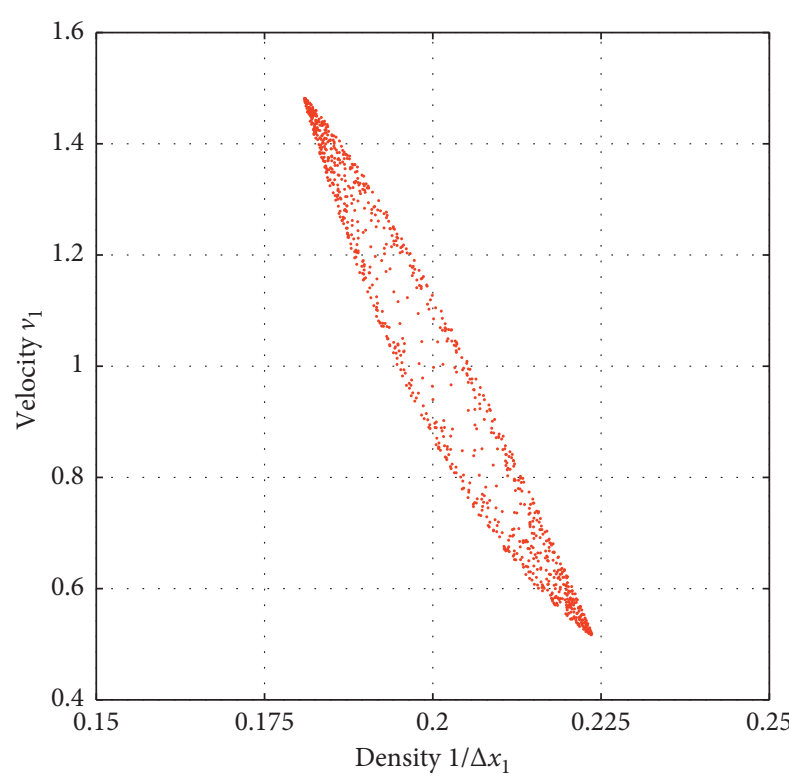

(a)

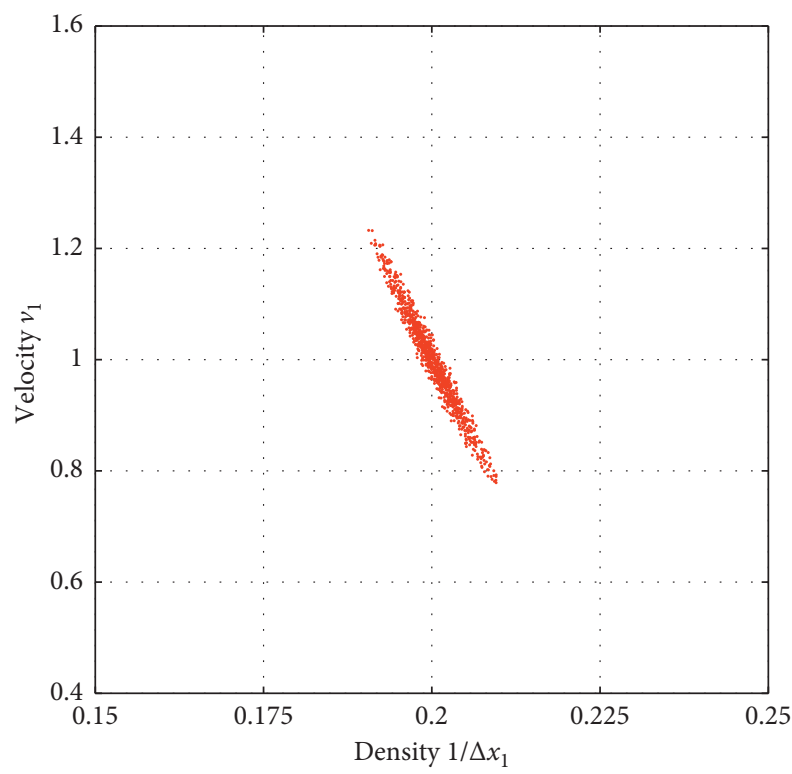

(c)

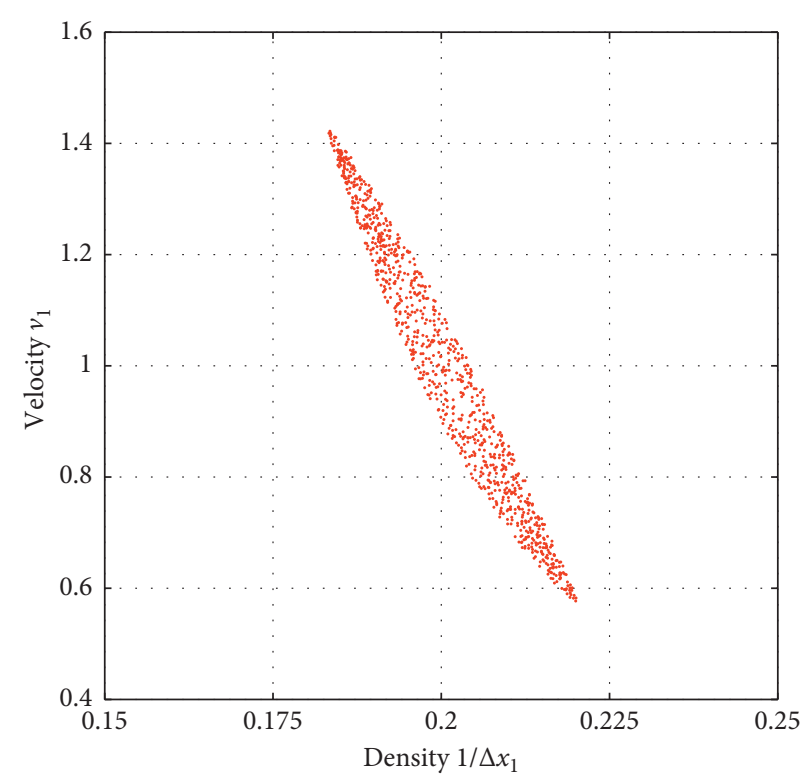

(b)

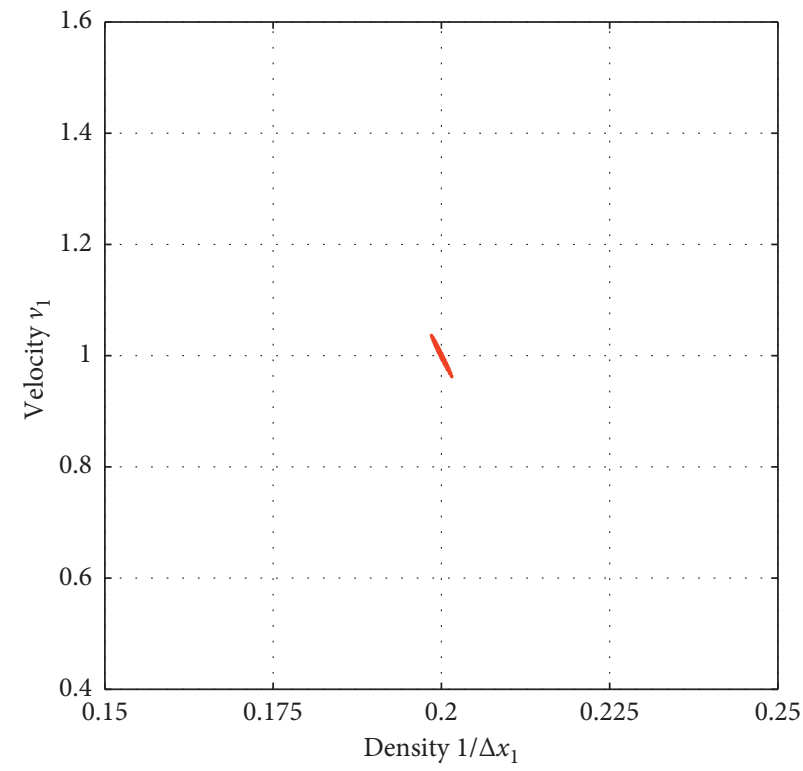

(d)

Figure 7: The hysteresis loop for different $\lambda_{1}$ under $\lambda_{2}=0, p=0$, and $\tau=1$. (a) $\lambda_{1}=0$ and $\lambda_{2}=0$, (b) $\lambda_{1}=0.01$ and $\lambda_{2}=0$, (c) $\lambda_{1}=0.02$ and $\lambda_{2}=0$, and (d) $\lambda_{1}=0.03$ and $\lambda_{2}=0$.

gain the following formula omitting the terms of $i k$ of which the order number exceeds two:

$$
\begin{aligned}
& {\left[\alpha V^{\prime}(h)+\left(\lambda_{2}-\alpha\right) z_{1}-\left(\lambda_{1}+\lambda_{2}\right)(1+p) z_{1}\right](i k)} \\
& \quad+\left[\frac{1}{2} \alpha V^{\prime}(h)+\left(\lambda_{2}-\alpha\right) z_{2}-z_{1}^{2}+\left(\lambda_{1}+\lambda_{2}\right)(1+p) z_{1}^{2} \tau\right. \\
& \left.\quad-\left(\lambda_{1}+\lambda_{2}\right)(1+p) z_{2}\right](i k)^{2}=0 .
\end{aligned}
$$

Then, there exists

$$
\left\{\begin{array}{l}
\alpha V^{\prime}(h)+\left(\lambda_{2}-\alpha\right) z_{1}-\left(\lambda_{1}+\lambda_{2}\right)(1+p) z_{1}=0 \\
\frac{1}{2} \alpha V^{\prime}(h)+\left(\lambda_{2}-\alpha\right) z_{2}-z_{1}^{2}+\left(\lambda_{1}+\lambda_{2}\right)(1+p) z_{1}^{2} \tau \\
\quad-\left(\lambda_{1}+\lambda_{2}\right)(1+p) z_{2}=0 .
\end{array}\right.
$$

Following that, we get the value of $z_{1}$ and $z_{2}$ by calculating (10) as 


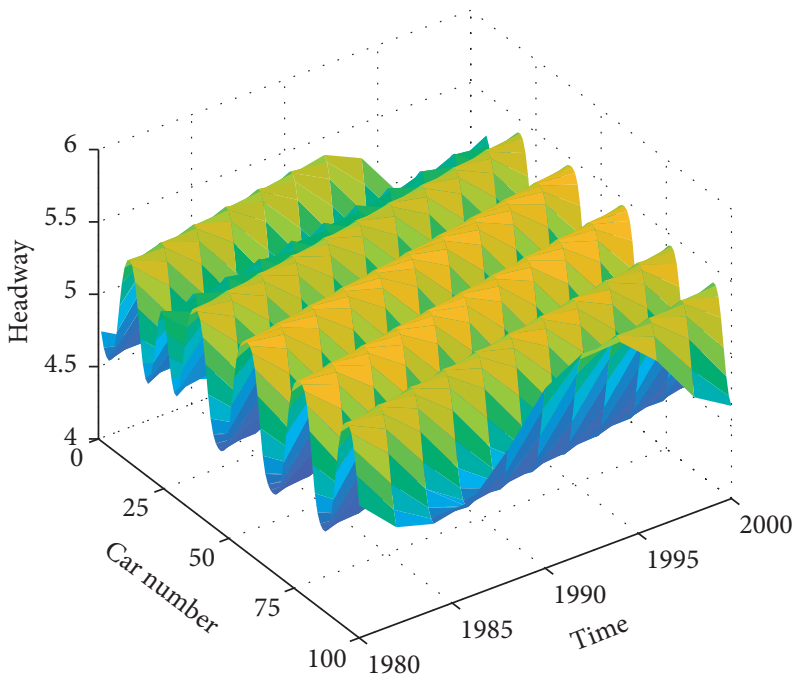

(a)

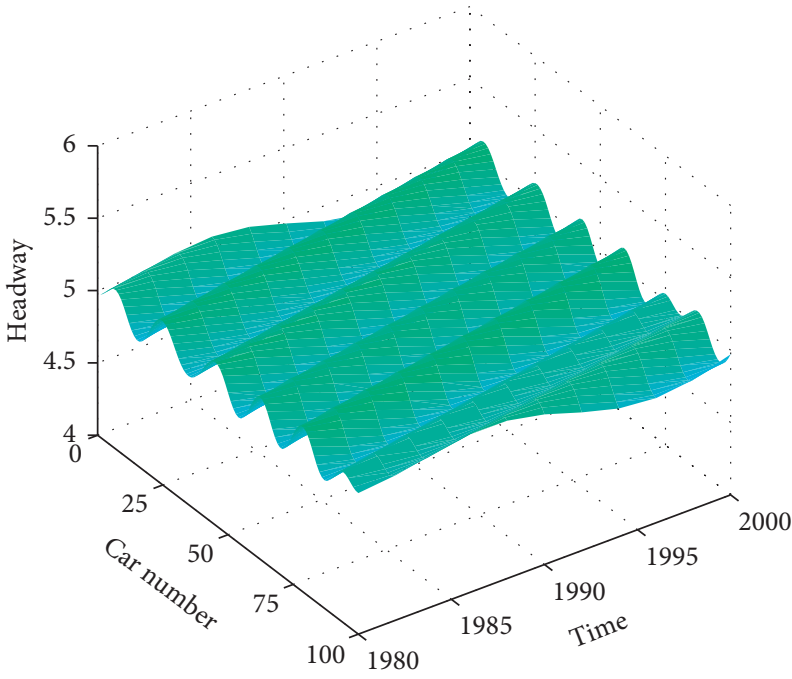

(c)

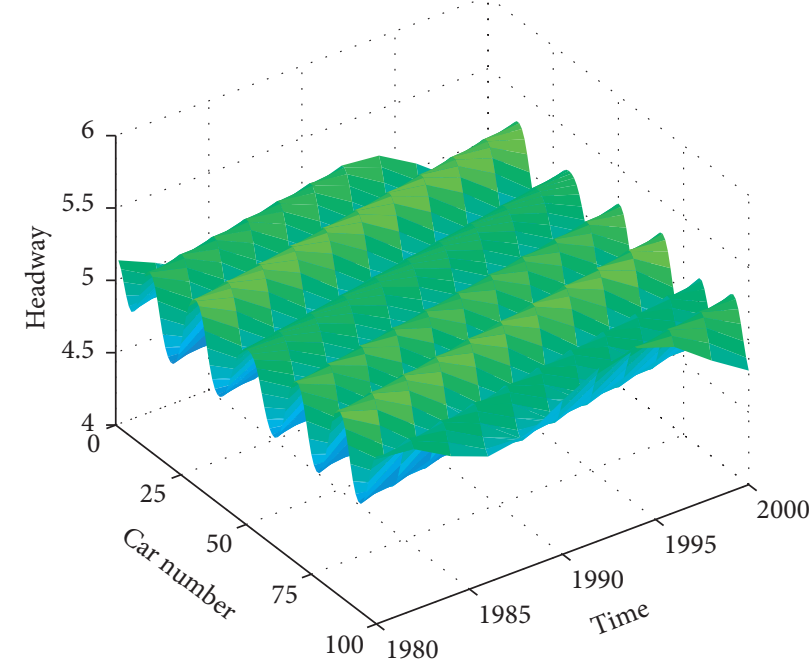

(b)

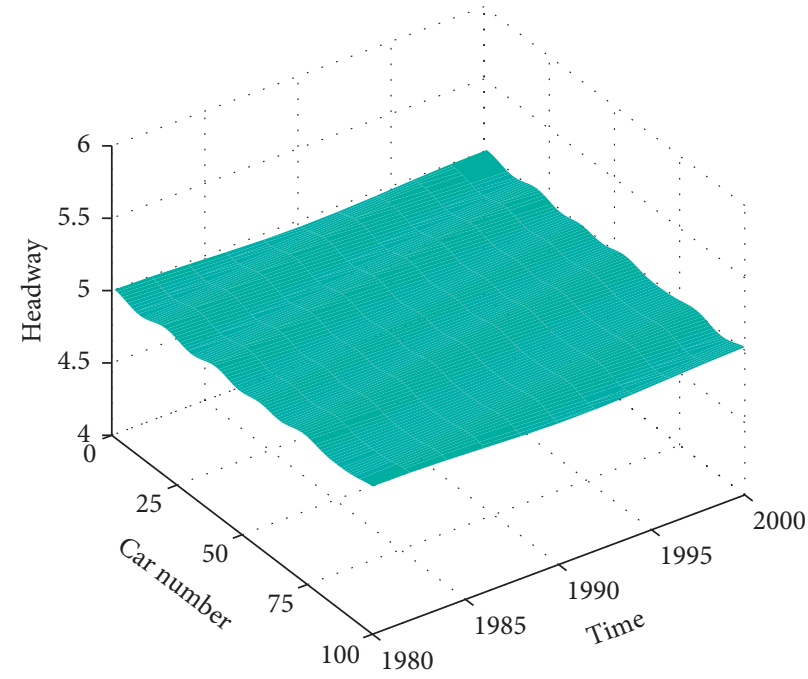

(d)

FIGURE 8: The profiles of headway for different $\lambda_{2}$ under $\lambda_{1}=0.01, p=0$, and $\tau=1$. (a) $\lambda_{1}=0.01$ and $\lambda_{2}=0$, (b) $\lambda_{1}=0.01$ and $\lambda_{2}=0.05$, (c) $\lambda_{1}=0.01$ and $\lambda_{2}=0.07$, and (d) $\lambda_{1}=0.01$ and $\lambda_{2}=0.1$.

$$
\left\{\begin{array}{l}
z_{1}=\frac{\alpha V^{\prime}(h)}{\lambda_{1}+\left(\lambda_{1}+\lambda_{2}\right) p+\alpha}, \\
z_{2}=\frac{\left[\left(\lambda_{1}+\lambda_{2}\right)(1+p) \tau-1\right] \alpha^{2}\left[V^{\prime}(h)\right]^{2}+(1 / 2) \alpha\left[\lambda_{1}+\left(\lambda_{1}+\lambda_{2}\right) p+\alpha\right]^{2}}{\left[\lambda_{1}+\left(\lambda_{1}+\lambda_{2}\right) p+\alpha\right]^{3}} .
\end{array}\right.
$$

The traffic congestion will be avoidable when $z_{2}$ is positive. Thus, we can get the linear stability criterion as

$$
\left[\left(\lambda_{1}+\lambda_{2}\right)(1+p) \tau-1\right] \alpha V^{\prime}(h)+\frac{1}{2}\left[\lambda_{1}+\left(\lambda_{1}+\lambda_{2}\right) p+\alpha\right]^{2}>0 .
$$

From (12), we can obtain the neutral stability curve which can be shown as

$$
\alpha_{c}=-\left[A+V^{\prime}(h) B\right]+\sqrt{2 V^{\prime}(h) A B+\left[V^{\prime}(h)\right]^{2} B^{2}-3 A^{2}}
$$

where $A=\lambda_{1}+\left(\lambda_{1}+\lambda_{2}\right) p$ and $B=\left(\lambda_{1}+\lambda_{2}\right)(1+p) \tau-1$.

From (13), when $\lambda_{1}=\lambda_{2}=0$, the novel model reduces to the OVM, which is in accordance with the results of OVM.

In order to discuss the influences of the driver's desire for smooth driving, the self-stabilizing control effect, the velocity uncertainty, and the historical time gap on traffic flow, 


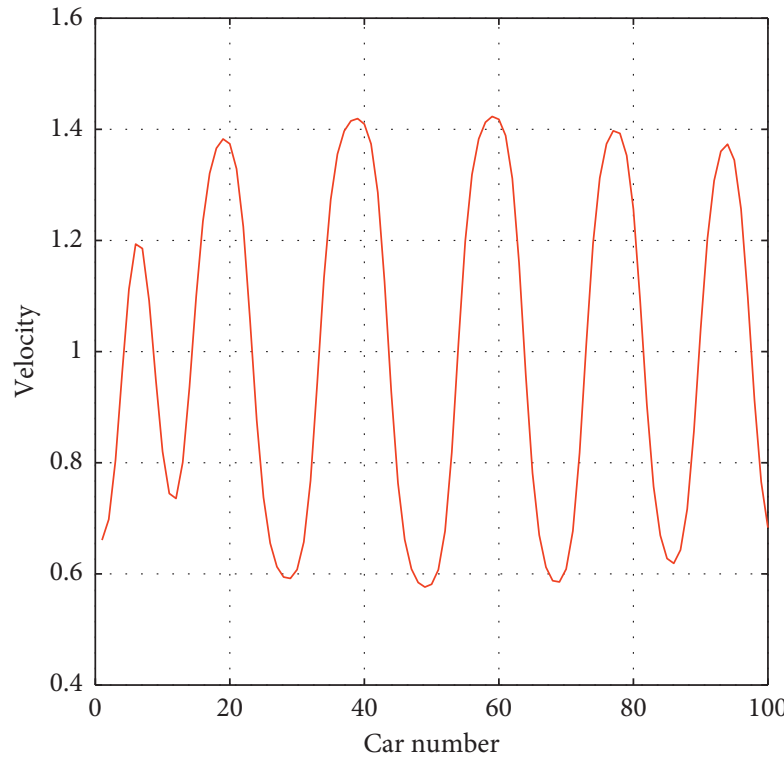

(a)

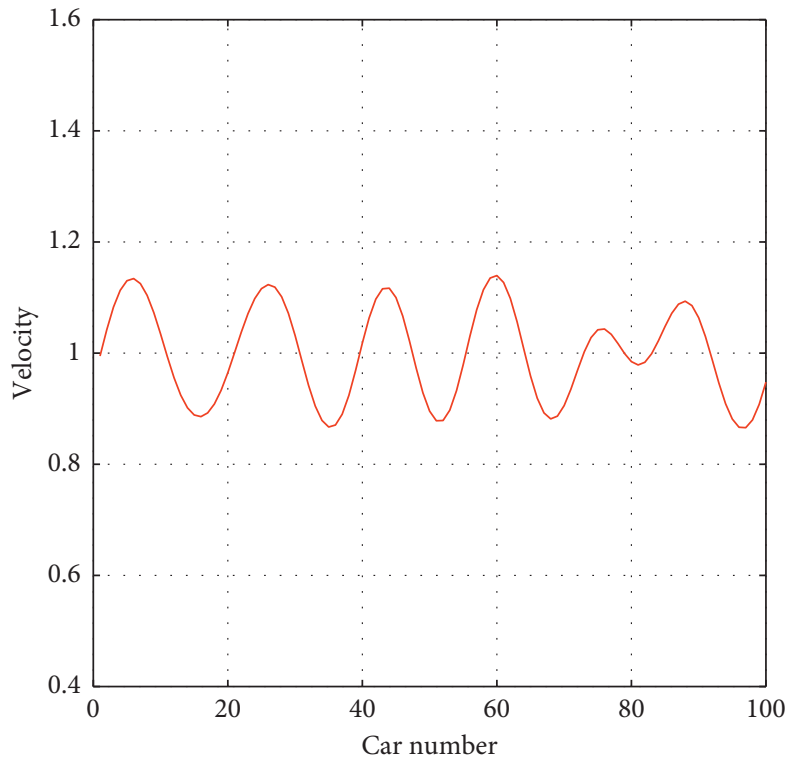

(c)

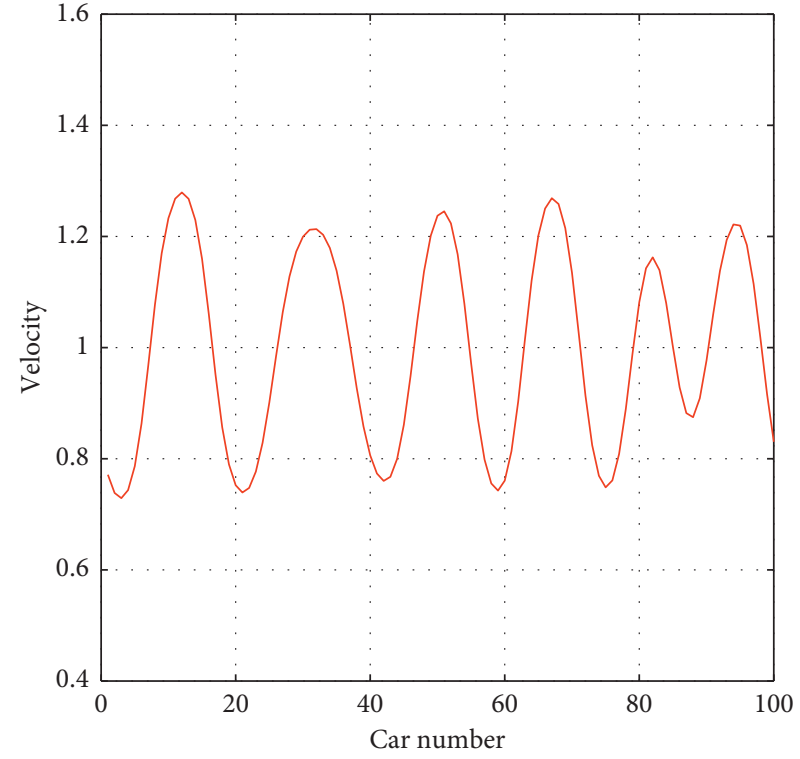

(b)

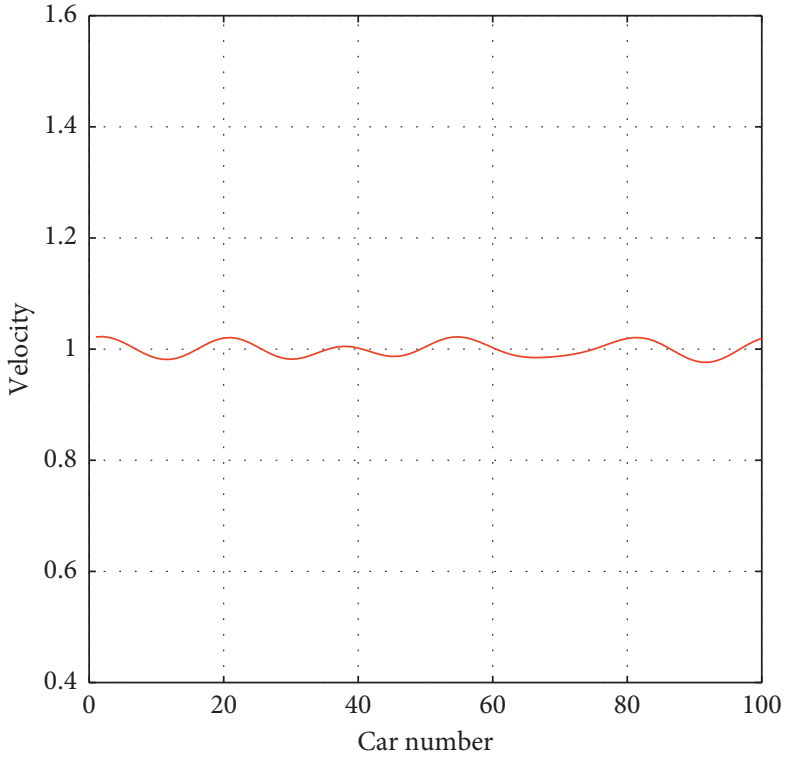

(d)

FiguRE 9: The profiles of velocity for different $\lambda_{2}$ under $\lambda_{1}=0.01, p=0$, and $\tau=1$. (a) $\lambda_{1}=0.01$ and $\lambda_{2}=0$, (b) $\lambda_{1}=0.01$ and $\lambda_{2}=0.05$, (c) $\lambda_{1}=0.01$ and $\lambda_{2}=0.07$, and (d) $\lambda_{1}=0.01$ and $\lambda_{2}=0.1$.

the neutral stability lines for different parameters are drawn in Figures 1-4.

For given neutral stability curves, the region below the line is the unstable region where the traffic jam will show up with a small derivation. On the contrary, the region above the line is the stable region where the traffic congestion will never occur when the outside perturbation is imposed on traffic flow. Based on this, we conduct the following analyses.

In Figure 1, the solid line is the neutral stability line of OVM with $\lambda_{1}=0$ and $\lambda_{2}=0$. When the value of $\lambda_{1}$ increases with fixed coefficients $\tau=1, \lambda_{2}=0$, and $p=0$, the height of lines will decrease which means the stable region becomes bigger. In other words, if the effect of the driver's desire for smooth driving is considered, the traffic flow becomes more stable by increasing the value of $\lambda_{1}$. Then in Figure 2, the fixed parameters are $\tau=1, \lambda_{1}=0.01$, and $p=0$; factors including the driver's desire for smooth driving and the self-stabilizing control are both considered. With increasing $\lambda_{2}$ from 0 to 0.08 , the stable region will increase little by little. Namely, the traffic flow becomes more stable when two effects are considered. From Figure 3, one can note that the stable region will also become bigger when the historical time gap $\tau$ increases. Finally, in Figure 4, we begin to discuss the influence of velocity uncertainty on traffic flow and the fixed parameters 


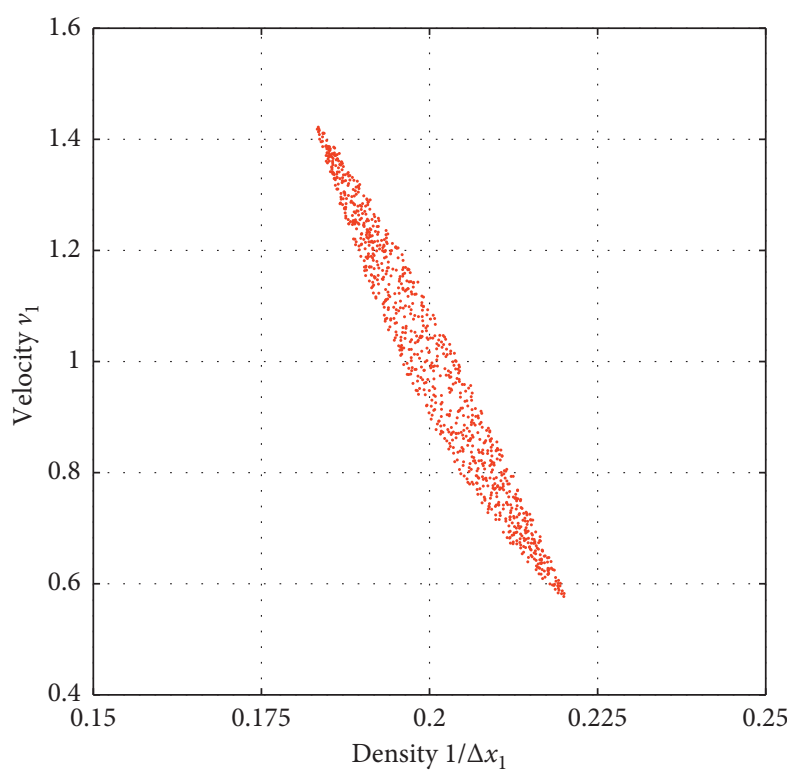

(a)

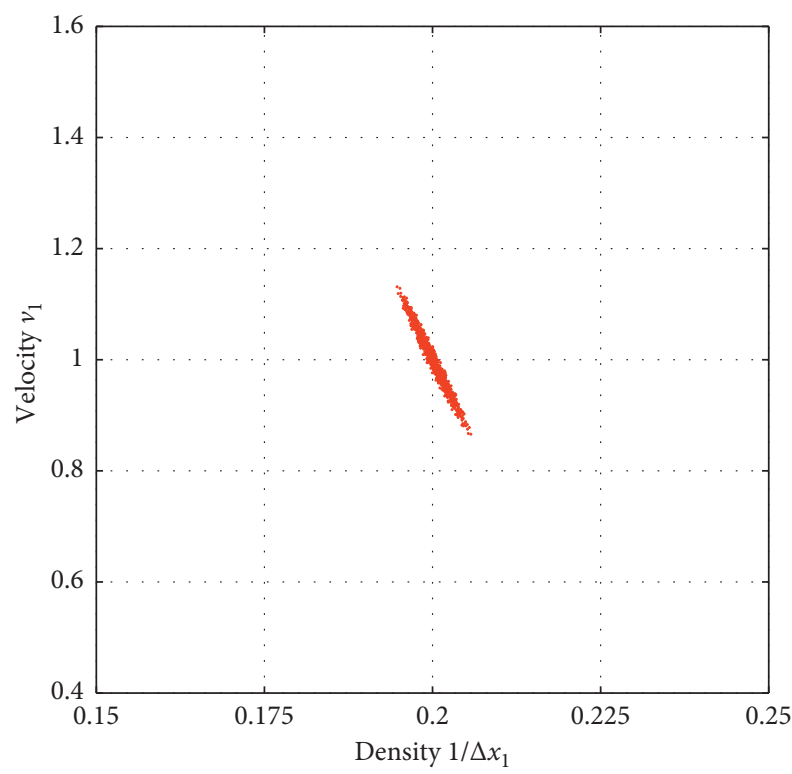

(c)

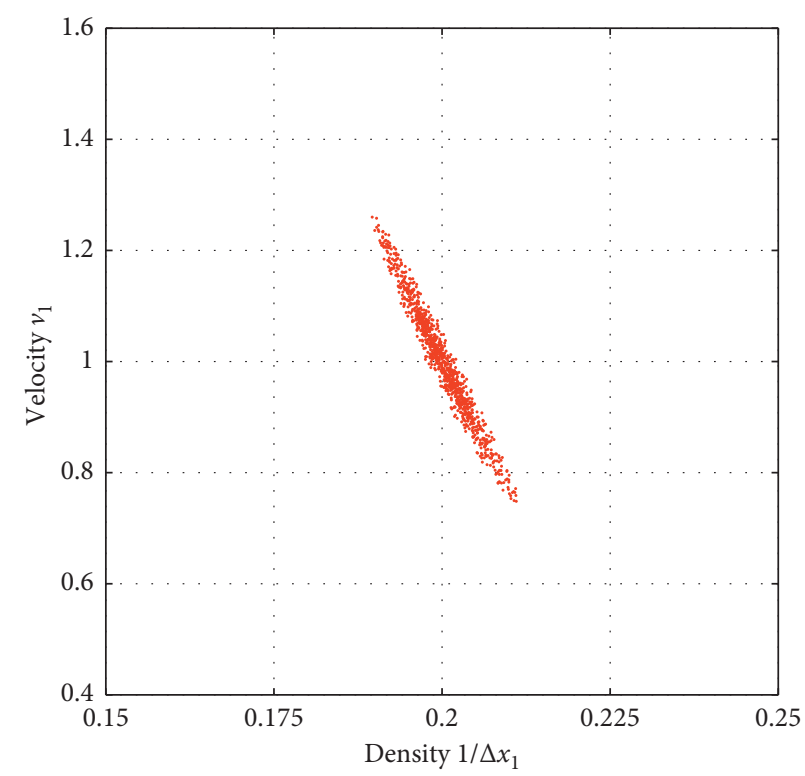

(b)

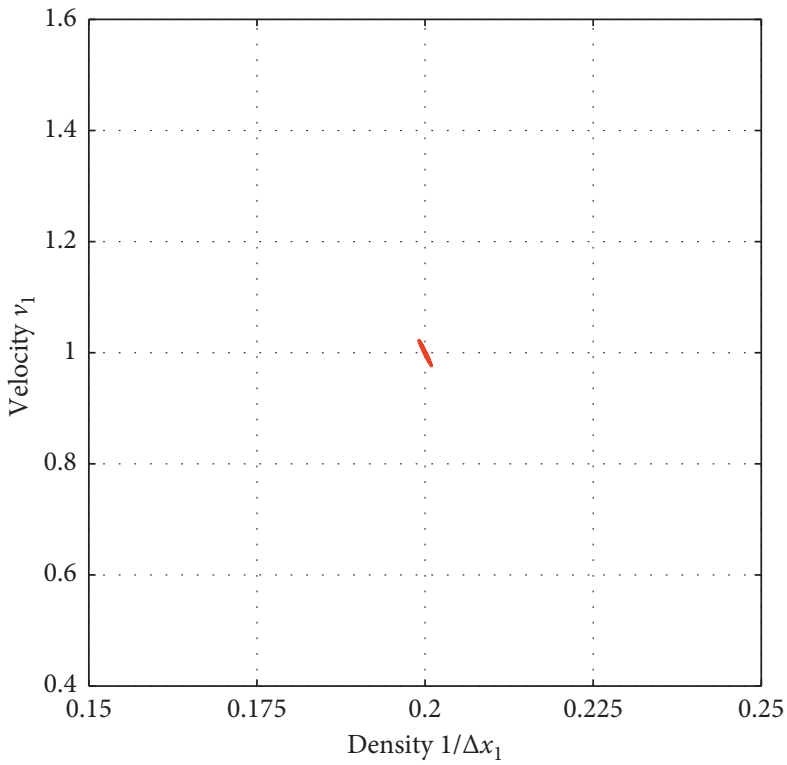

(d)

Figure 10: The hysteresis loop for different $\lambda_{2}$ under $\lambda_{1}=0.01, p=0$, and $\tau=1$. (a) $\lambda_{1}=0.01$ and $\lambda_{2}=0$, (b) $\lambda_{1}=0.01$ and $\lambda_{2}=0.05$, (c) $\lambda_{1}=0.01$ and $\lambda_{2}=0.07$, and (d) $\lambda_{1}=0.01$ and $\lambda_{2}=0.1$.

are $\lambda_{1}=0.01, \lambda_{2}=0.02$, and $\tau=1$. When $p=-0.25$, the driver overestimates the driving condition and increases the acceleration of vehicle, which may cause the appearance of traffic accident, so the unstable region is larger. When $p=0$, the historical velocity perceived by driver equals the real velocity. When $p=0.25$ and $p=0.45$, the driver underestimates the driving condition and makes a deceleration decision. One can notice that the stable region is larger with increasing the value of $p$, whereas the stability in this situation is "fictitious" at the price of inferior traffic efficiency, as can be confirmed as follows.

To sum up, in view of the figures of neutral stability curves and corresponding analyses, it is clear that the factors considered in the new model play a significant role in influencing the traffic flow's stability.

\section{Nonlinear Analysis}

The $\mathrm{mKdV}$ equation is inferred to describe the varying behaviors of traffic flow for critical point $\left(h_{c}, \alpha_{c}\right)$ in unstable region [45]. At first, define the slow variables $T$ and $X$ as

$$
\begin{aligned}
& T=\varepsilon^{3} t, \\
& X=\varepsilon(n+b t),
\end{aligned}
$$




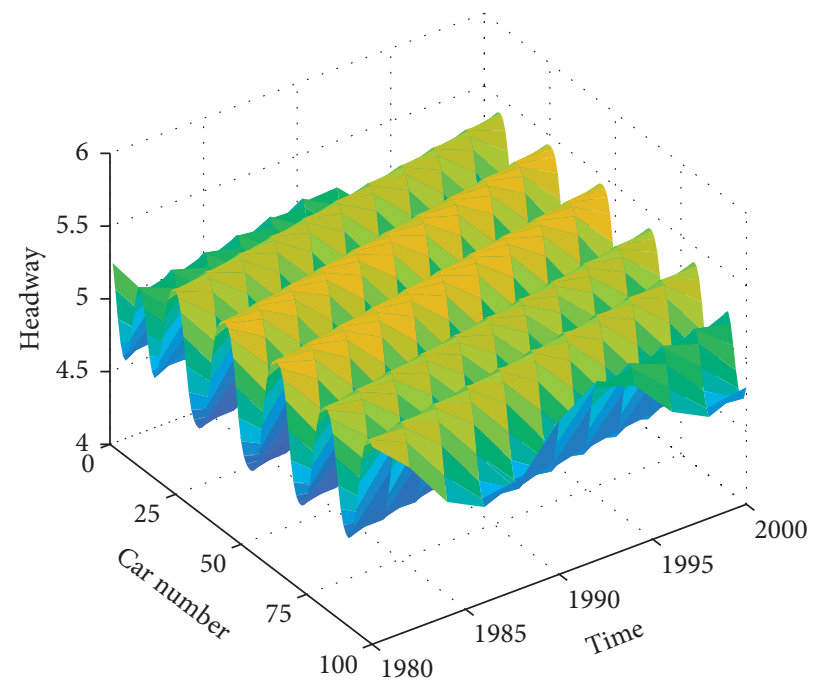

(a)

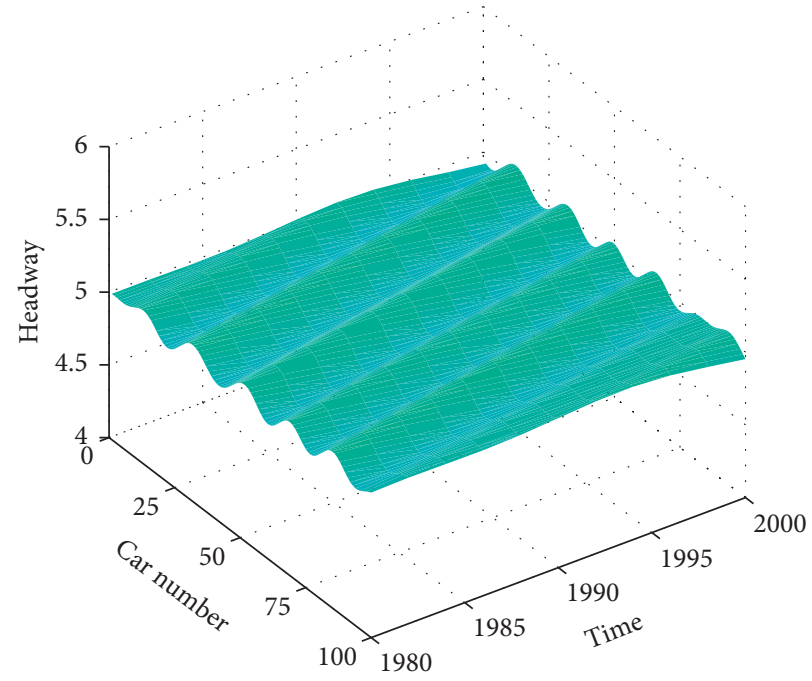

(c)

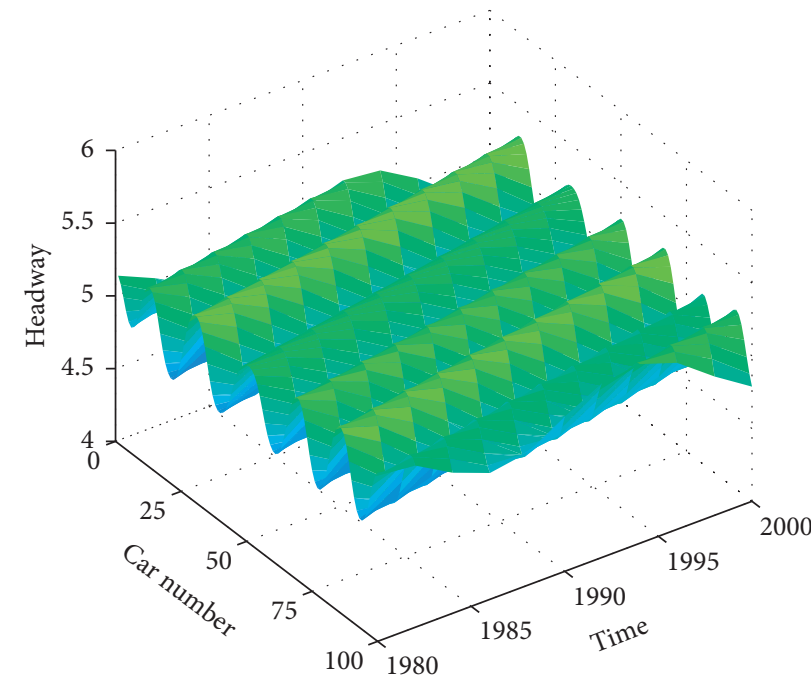

(b)

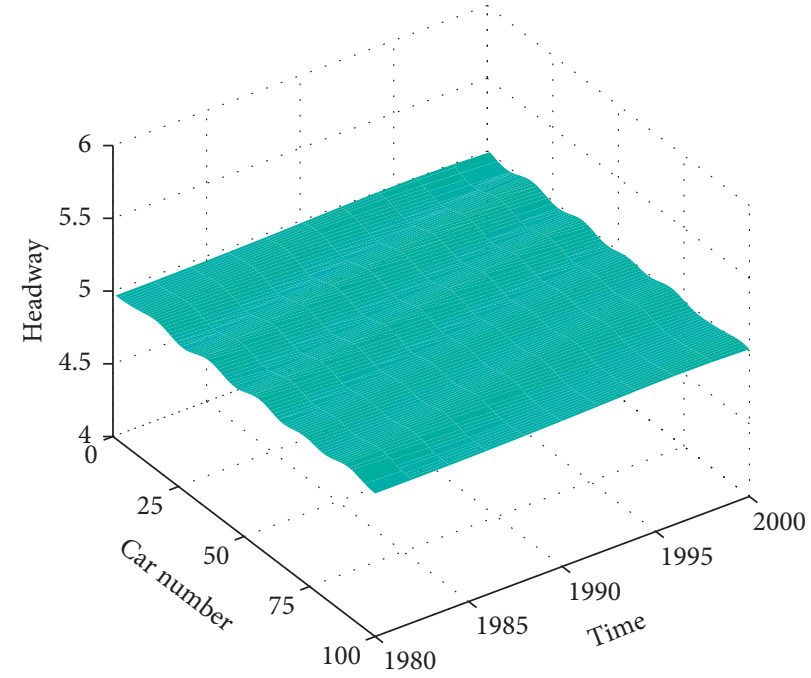

(d)

FIgURe 11: The profiles of headway for different $p$ under $\lambda_{1}=0.01, \lambda_{2}=0.05$, and $\tau=1$. (a) $p=-0.25$, (b) $p=0$, (c) $p=0.25$, and (d) $p=0.45$.

where $0<\varepsilon \ll 1$ and $b$ is an unknown constant which can be calculated.

The space headway distance $\Delta x_{n}(t)$ with a disturbance will be expressed ast

$$
\Delta x_{n}(t)=h_{c}+\varepsilon R(X, T) .
$$

Inserting (14) and (15) into (4) and extending to the fifth order of $\varepsilon$, we will obtain

$$
\begin{aligned}
& {\left[\alpha V^{\prime}+\left(\lambda_{2}-\alpha\right) b-\Omega b\right] \varepsilon^{2} \partial_{X} R+\left[\frac{1}{2} \alpha V^{\prime}+b^{2}+\Omega b^{2} \tau\right] \varepsilon^{3} \partial_{X}^{2} R} \\
& +\left\{\left[\frac{1}{6} \alpha V^{\prime}+\frac{1}{2} \Omega b^{3} \tau^{2}\right] \partial_{X}^{3} R+\frac{1}{6} \alpha V^{\prime \prime \prime} \partial_{X} R^{3}-\left[\left(\lambda_{2}-\alpha\right)-\Omega\right] \partial_{T} R\right\} \varepsilon^{4} \\
& \quad+\left\{\left[\frac{1}{24} \alpha V^{\prime}+\frac{1}{6} \Omega b^{4} \tau^{3}\right] \partial_{X}^{4} R+\frac{1}{12} \alpha V^{\prime \prime \prime} \partial_{X}^{2} R^{3}+(2 \Omega b \tau-2 b) \partial_{X T} R\right\} \varepsilon^{5}=0,
\end{aligned}
$$

where $\partial_{X}^{k} R=\partial^{k} R / \partial X^{k}, \partial_{X T} R=\partial^{2} R / \partial X \quad \partial T, \partial_{X} R=\partial R / \partial X$, $V^{\prime}=\left.(\partial V(h) / \partial h)\right|_{h=h_{c}}, R=R(X, T), V^{\prime \prime \prime}=\left.\left(\partial^{3} V(h) / \partial h^{3}\right)\right|_{h=h_{c}}$, and $\Omega=\left(\lambda_{1}+\lambda_{2}\right)(1+p)$.
Taking $b=\alpha V^{\prime} /\left(\lambda_{1}+\left(\lambda_{1}+\lambda_{2}\right) p+\alpha\right)$ and $\alpha=\alpha_{c}\left(1-\varepsilon^{2}\right)$ into (16), then the coefficients of $\varepsilon^{2}$ and $\varepsilon^{3}$ can be eliminated; (16) will be translated into 


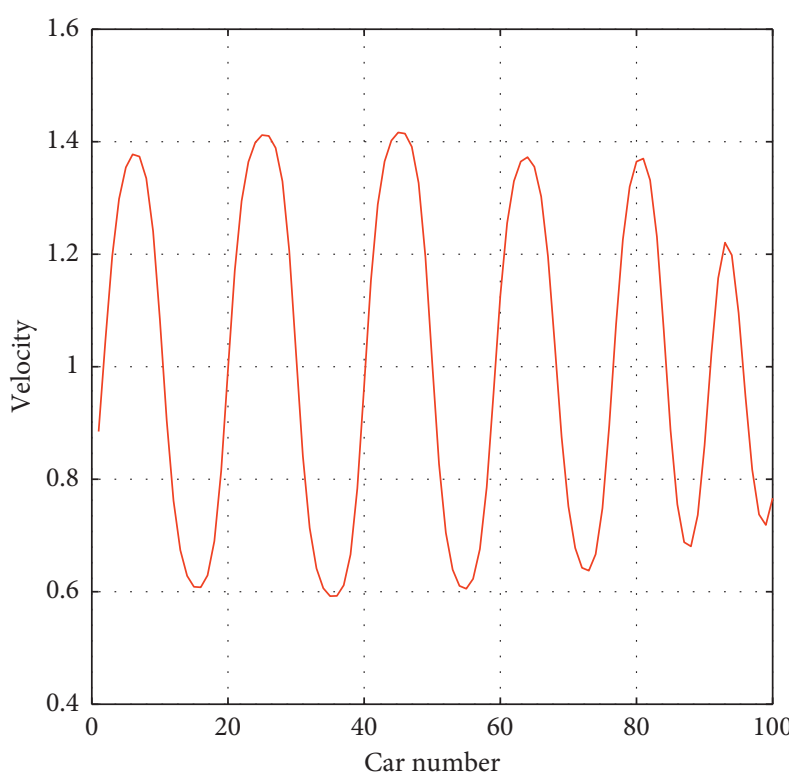

(a)

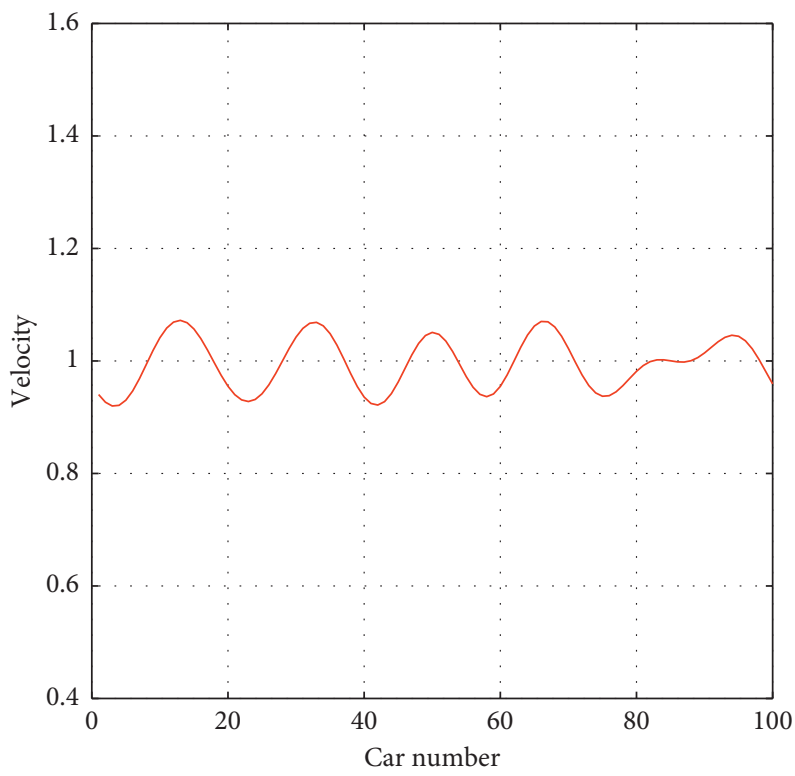

(c)

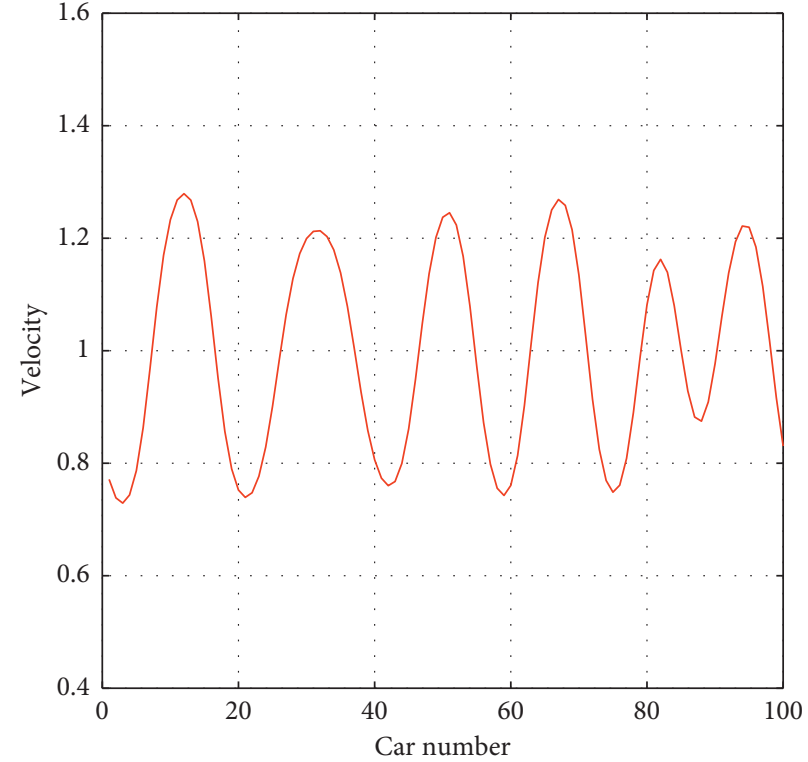

(b)

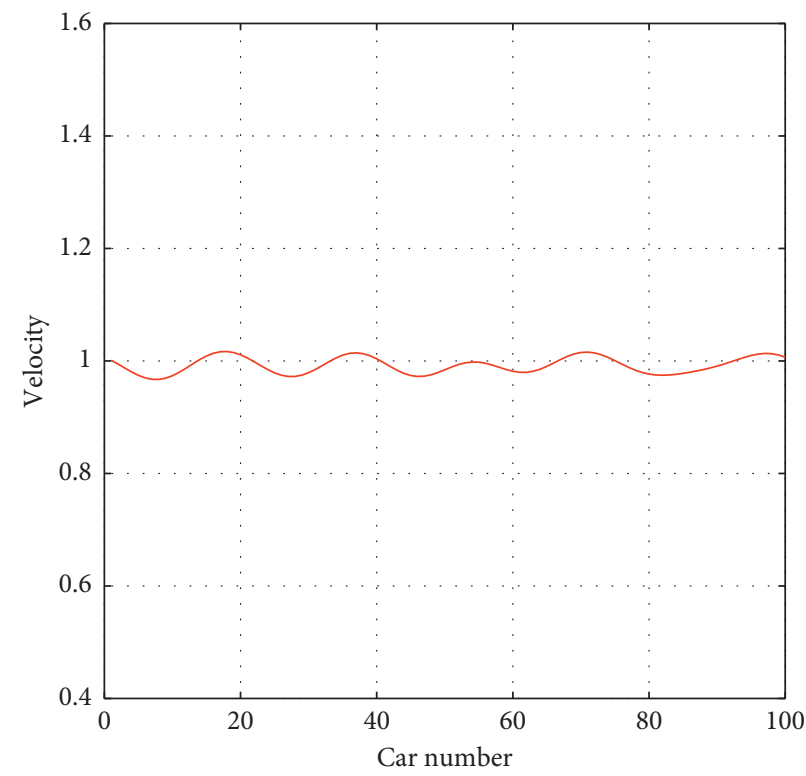

(d)

FIGURE 12: The profiles of velocity for different $p$ under $\lambda_{1}=0.01, \lambda_{2}=0.05$, and $\tau=1$. (a) $p=-0.25$, (b) $p=0$, (c) $p=0.25$, and (d) $p=0.45$.

$$
\begin{aligned}
& \left\{\left[\frac{1}{6} \alpha V^{\prime}+\frac{1}{2} \Omega b^{3} \tau^{2}\right] \partial_{X}^{3} R+\frac{1}{6} \alpha V^{\prime \prime \prime} \partial_{X} R^{3}-\left[\left(\lambda_{2}-\alpha\right)-\Omega\right] \partial_{T} R\right\} \varepsilon^{4} \\
& \quad+\left\{\left[\frac{1}{24} \alpha V^{\prime}+\frac{1}{6} \Omega b^{4} \tau^{3}\right] \partial_{X}^{4} R+\frac{1}{12} \alpha V^{\prime \prime \prime} \partial_{X} R^{3}+(2 \Omega b \tau-2 b) \partial_{X T} R+\frac{\left[\lambda_{1}+\left(\lambda_{1}+\lambda_{2}\right) p+\alpha\right]^{2}}{4[1-\Omega \tau]} \partial_{X}^{2} R\right\} \varepsilon^{5}=0 .
\end{aligned}
$$

Equation (17) can be simplified as follows:

$\varepsilon^{4}\left[\partial_{T} R-\xi_{1} \partial_{X}^{3} R+\xi_{2} \partial_{X} R^{3}\right]+\varepsilon^{5}\left[\xi_{3} \partial_{X}^{2} R+\xi_{4} \partial_{X}^{4} R+\xi_{5} \partial_{X}^{2} R^{3}\right]=0$,

where

$$
\xi_{1}=-\left[\frac{1}{6} \alpha V^{\prime}+\frac{1}{2}\left(\lambda_{1}+\lambda_{2}\right)(1+p) b^{3} \tau^{2}\right]
$$

$\xi_{2}=\frac{1}{6} \alpha V^{\prime \prime \prime}$ 


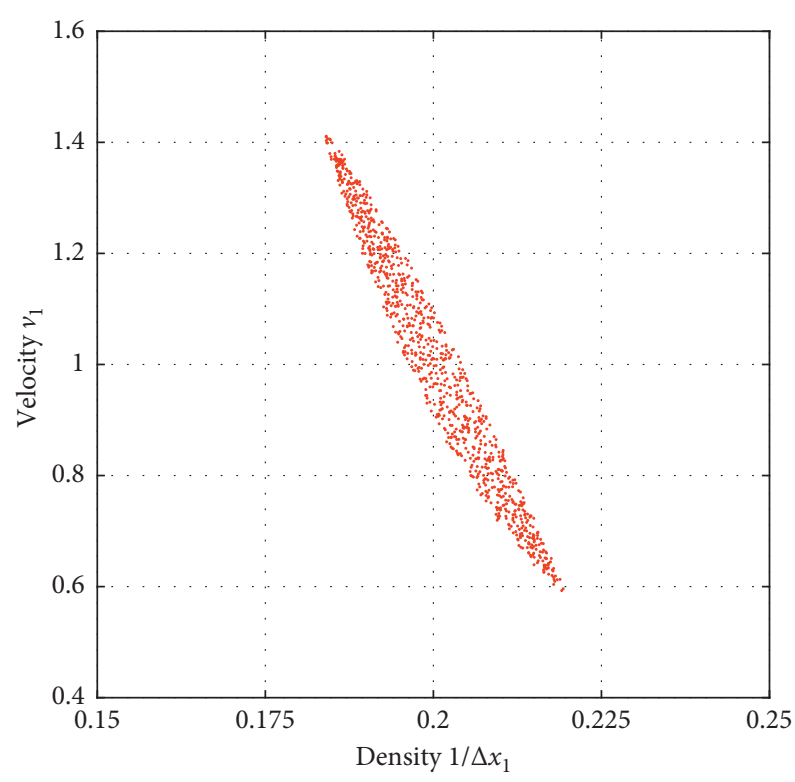

(a)

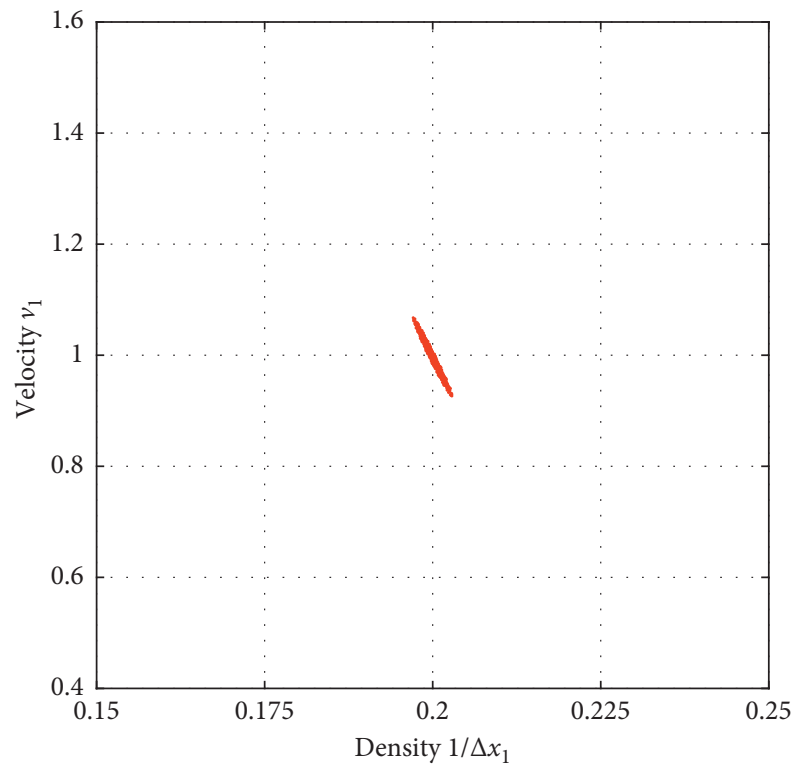

(c)

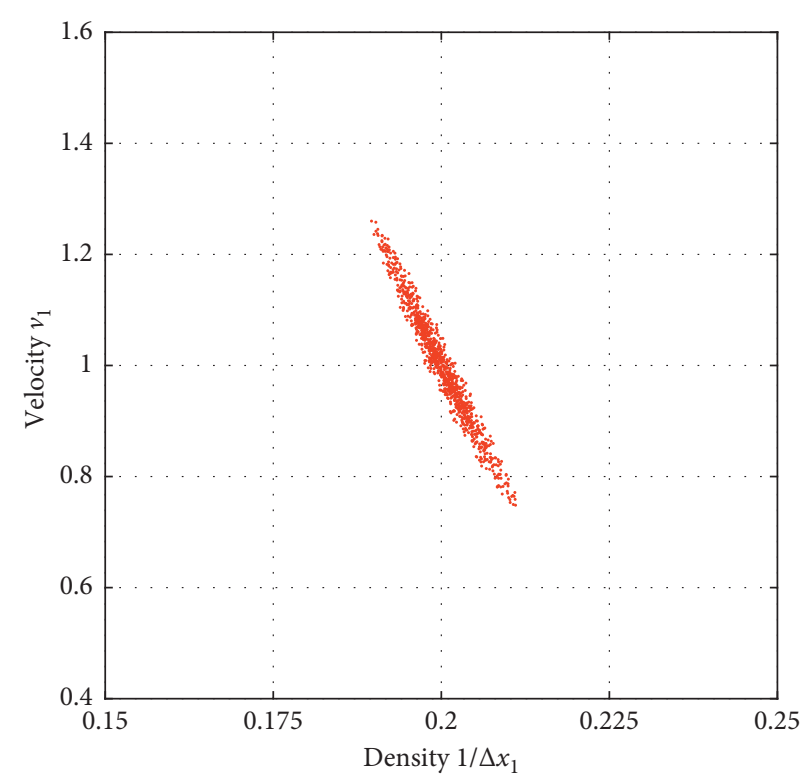

(b)

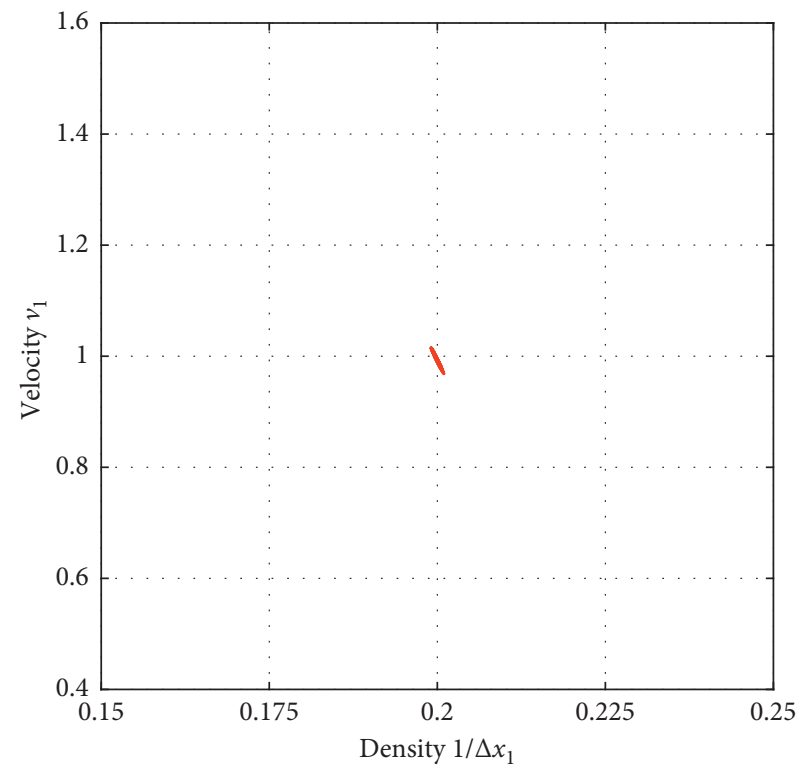

(d)

Figure 13: The hysteresis loop for different $p$ under $\lambda_{1}=0.01, \lambda_{2}=0.05$, and $\tau=1$. (a) $p=-0.25$, (b) $p=0$, (c) $p=0.25$, and (d) $p=0.45$.

$$
\begin{aligned}
& \xi_{3}=\frac{\left[\lambda_{1}+\left(\lambda_{1}+\lambda_{2}\right) p+\alpha\right]^{2}}{4\left[1-\left(\lambda_{1}+\lambda_{2}\right)(1+p) \tau\right]} \\
& \xi_{4}=\frac{1}{24} \alpha V^{\prime}+\frac{1}{6}\left(\lambda_{1}+\lambda_{2}\right)(1+p) b^{4} \tau^{3} \\
& \xi_{5}=\frac{1}{12} \alpha V^{\prime \prime \prime}
\end{aligned}
$$

Conducting the conversion of $T^{\prime}=\xi_{1} T$ and $R^{\prime}=\sqrt{\xi_{2} / \xi_{1}} R$, we will obtain the regularized $\mathrm{mKdV}$ equation with a correction term $o(\varepsilon)$ :

$$
\partial_{T^{\prime}} R^{\prime}-\partial_{X}^{3} R^{\prime}+\partial_{X} R^{\prime 3}+\varepsilon\left[\frac{\xi_{3}}{\xi_{1}} \partial_{X}^{2} R^{\prime}+\frac{\xi_{4}}{\xi_{1}} \partial_{X}^{4} R^{\prime}+\frac{\xi_{5}}{\xi_{2}} \partial_{X}^{2} R^{\prime 3}\right]=0 .
$$

Ignoring the term $o(\varepsilon)$, we can get a soliton solution of (24) which can be expressed as

$$
R_{0}^{\prime}\left(X, T^{\prime}\right)=\sqrt{c} \tanh \left[\sqrt{\frac{c}{2}}\left(X-c T^{\prime}\right)\right] .
$$

Supposing $\quad R^{\prime}\left(X, T^{\prime}\right)=R_{0}^{\prime}\left(X, T^{\prime}\right)+\varepsilon R_{1}^{\prime}\left(X, T^{\prime}\right), \quad$ only when solvability condition is fulfilled, we can gain the value of $c$; the solvability condition is 


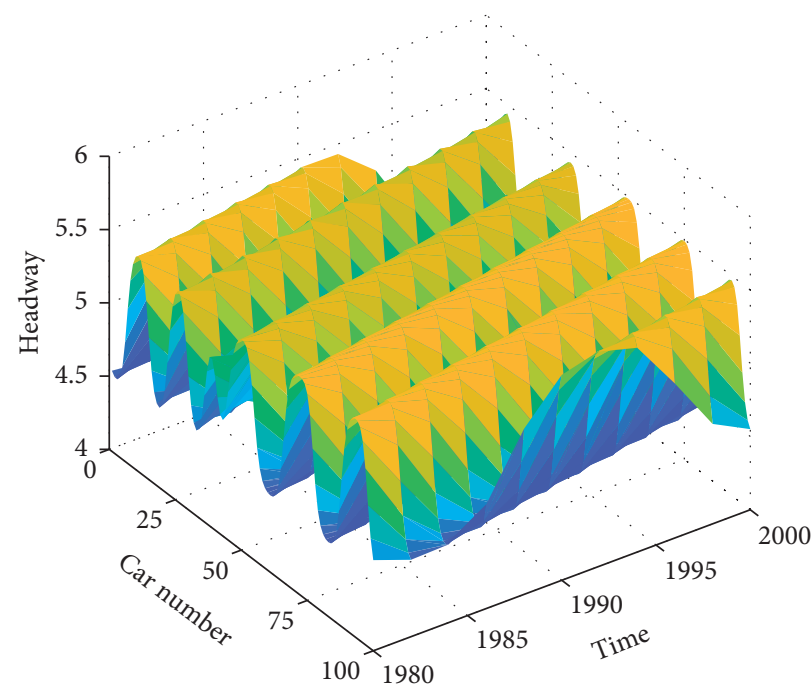

(a)

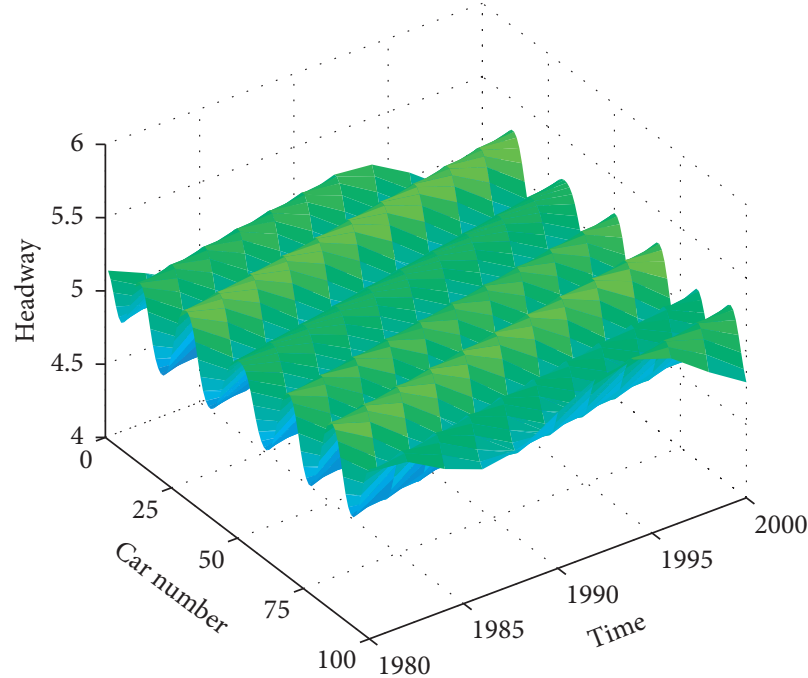

(c)

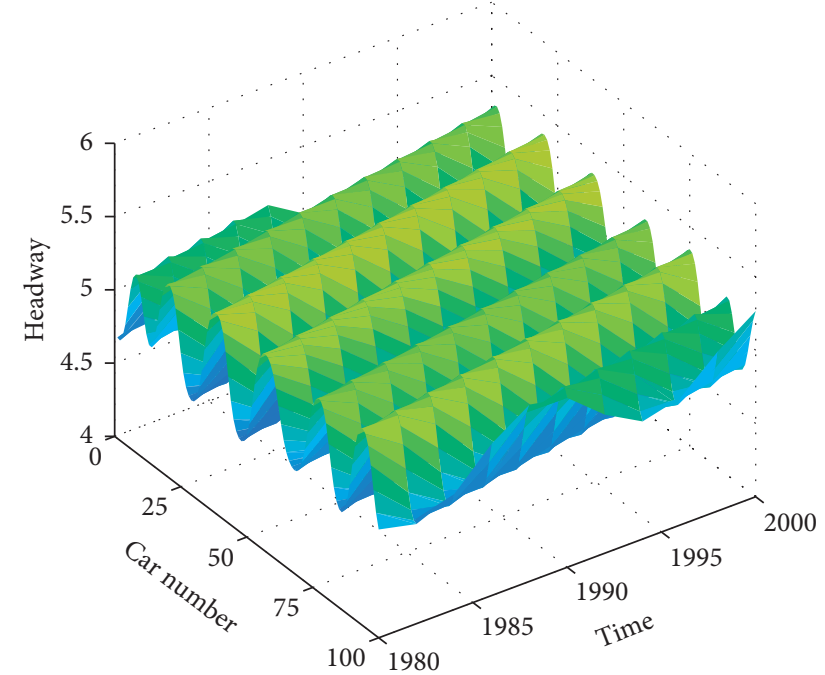

(b)

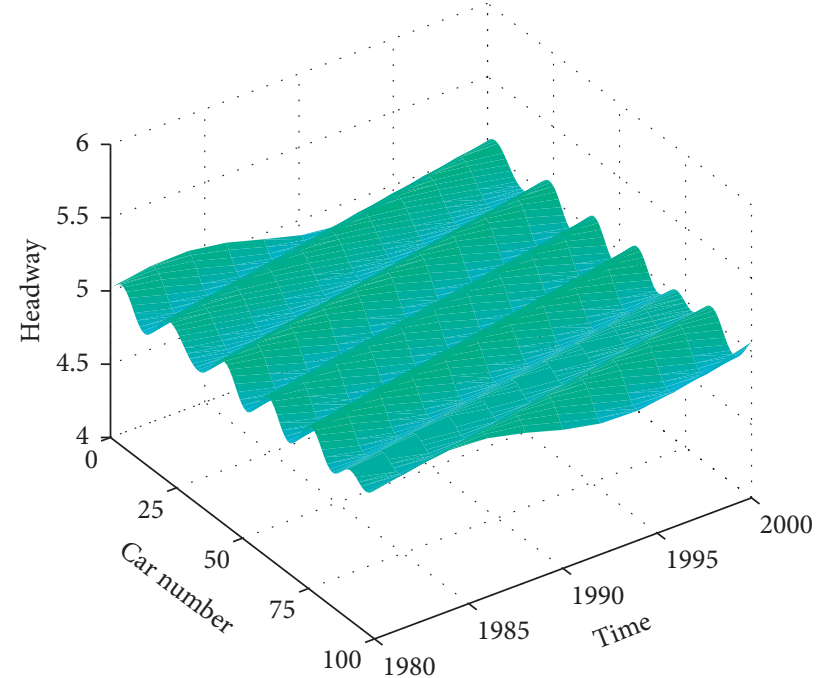

(d)

Figure 14: The profiles of headway for different $\tau$ under $\lambda_{1}=0.01, \lambda_{2}=0.05$, and $p=0$. (a) $\tau=0$, (b) $\tau=0.75$, (c) $\tau=1$, and (d) $\tau=1.5$.

$$
\left(R_{0}^{\prime}, M\left[R_{0}^{\prime}\right]\right) \equiv \int_{-\infty}^{+\infty} \mathrm{dX} R_{0}^{\prime} M\left[R_{0}^{\prime}\right]=0
$$

where $M\left[R_{0}^{\prime}\right]=\left(\xi_{3} / \xi_{1}\right) \partial_{X}^{2} R^{\prime}+\left(\xi_{4} / \xi_{1}\right) \partial_{X}^{4} R^{\prime}+\left(\xi_{5} / \xi_{2}\right) \partial_{X}^{2} R^{\prime 3}$.

Based on the solvability condition above, we can get the propagation speed $c$ :

$$
c=\frac{5 \xi_{2} \xi_{3}}{2 \xi_{2} \xi_{4}-3 \xi_{1} \xi_{5}} .
$$

Thus, the kink-antikink soliton solution of the space headway distance is given by

$$
\Delta x_{n}(t)=h_{c}+\sqrt{\frac{\xi_{1} c}{\xi_{2}}\left(\frac{\alpha}{\alpha_{c}}-1\right)} \times \tanh \left[\sqrt{\frac{c}{2}\left(\frac{\alpha}{\alpha_{c}}-1\right)} \times\left(n+\left(1-c \xi_{1}\right)\left(\frac{\alpha}{\alpha_{c}}-1\right) t\right)\right] .
$$

Here, the amplitude of traffic jam wave is

$$
A=\sqrt{\frac{c \xi_{1}}{\xi_{2}}\left(1-\frac{\alpha}{\alpha_{c}}\right)}
$$

One can note that the density wave can represent the property of traffic congestion, which is decided by the $\mathrm{mKdV}$ equation. From (19), (20), and (29), the parameters of factors considered in the new model have a momentous influence on influencing the amplitude of traffic jam wave. 


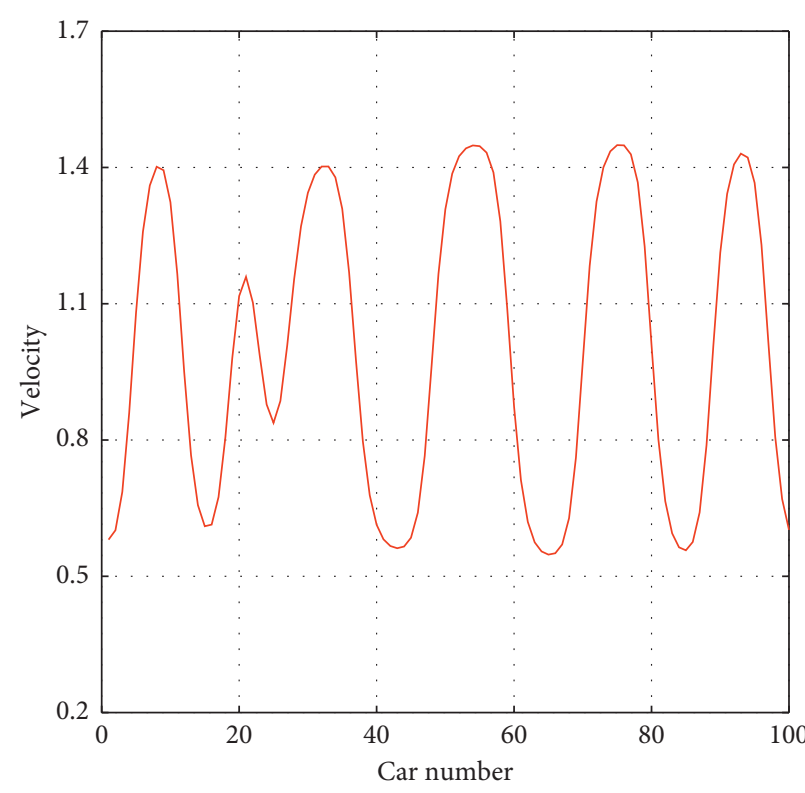

(a)

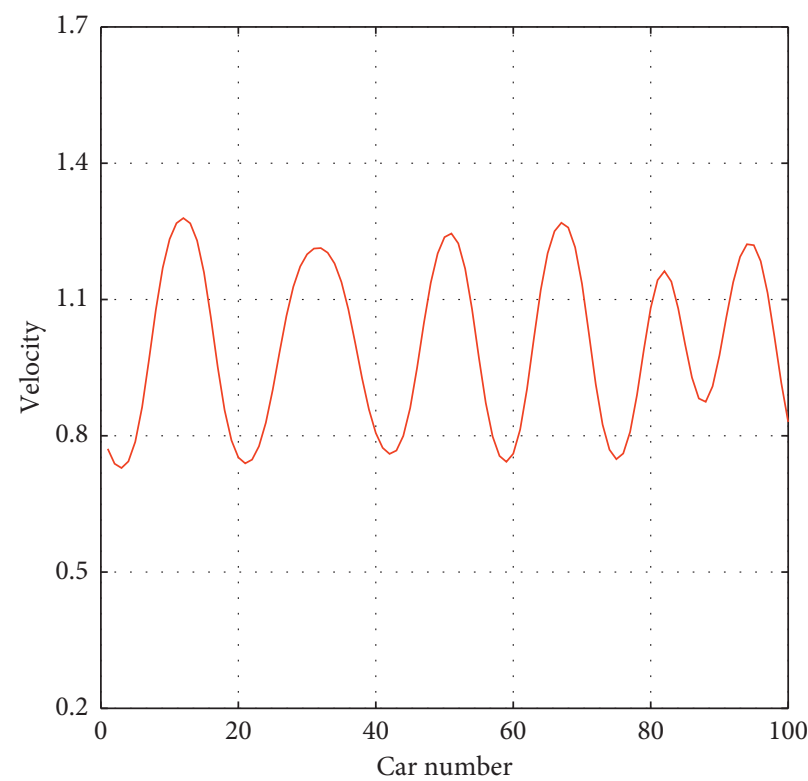

(c)

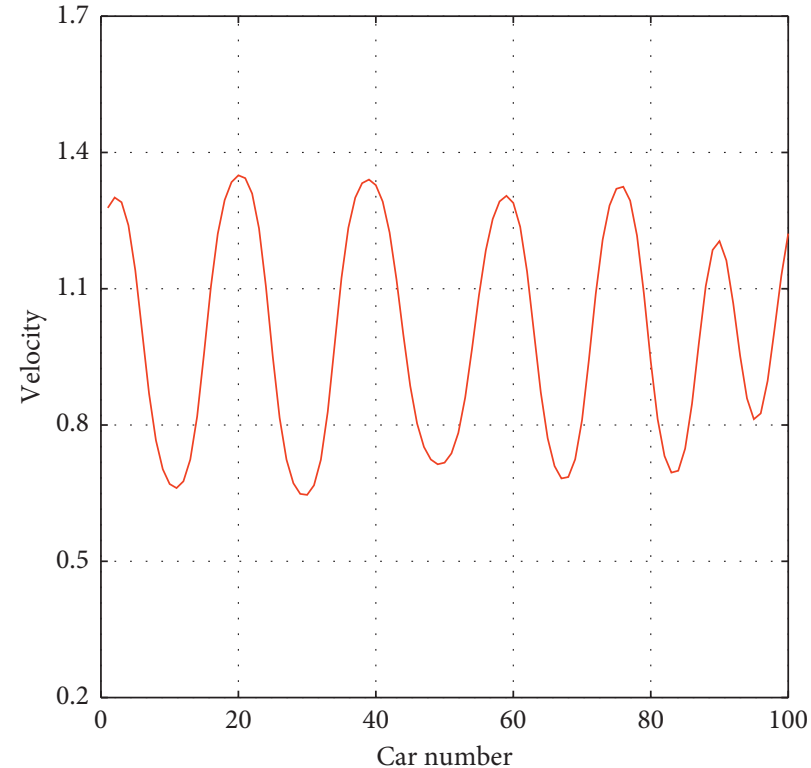

(b)

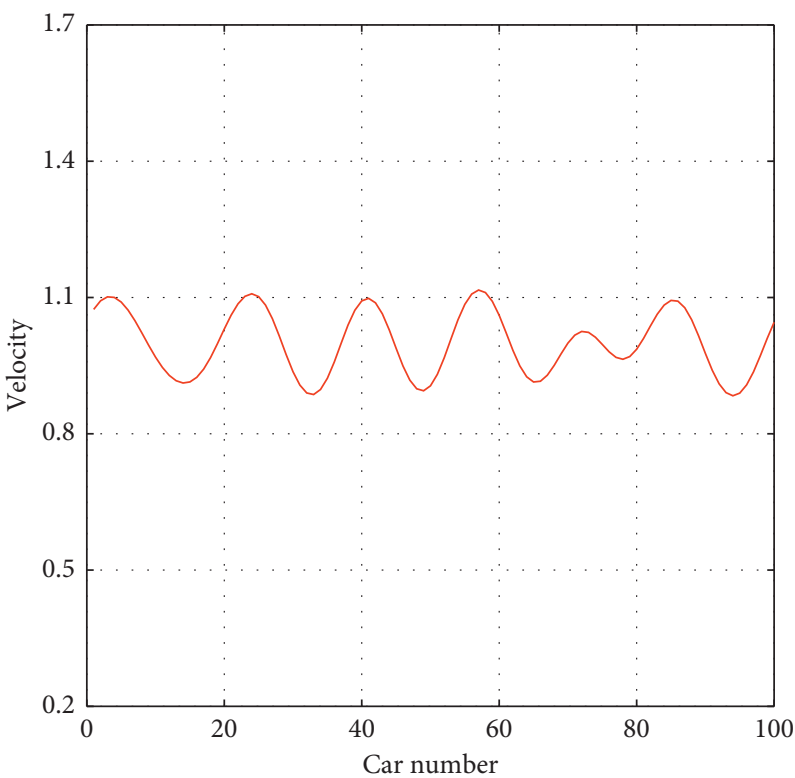

(d)

Figure 15: The profiles of velocity for different $\tau$ under $\lambda_{1}=0.01, \lambda_{2}=0.05$, and $p=0$. (a) $\tau=0$, (b) $\tau=0.75$, (c) $\tau=1$, and (d) $\tau=1.5$.

\section{Numerical Simulation}

Some simulation experiments are conducted for the extended model to verify the influence of factors considered on traffic flow in this part. At first, during the experiment, $N=$ 100 cars are running on the roadway and the relative fixed coefficients are set as follows:

$$
\begin{aligned}
v_{\max } & =2 \mathrm{~m} / \mathrm{s}, \\
\alpha & =1.7 \mathrm{~s}^{-1}, \\
h_{c} & =5 \mathrm{~m} .
\end{aligned}
$$

In addition, the initial conditions are set as follows:

$$
\begin{aligned}
\Delta x_{n}(\Delta t) & =\Delta x_{n}(0), \\
\Delta x_{n}(0) & = \begin{cases}h, & n \neq 50,51, \\
h-0.5, & n=50, \\
h+0.5, & n=51 .\end{cases}
\end{aligned}
$$

Figures $5-7$ are the headway profiles, the velocity profiles, and the hysteresis loop for different value of $\lambda_{1}$ with $\lambda_{2}=0, p=0$, and $\tau=1$, respectively. The hysteresis loop is the main reason that induces the traffic flow to be unstable state and causes the traffic phenomenon of stop and go. When $\lambda_{1}=0$, the novel model reduces to the OVM; Figures 5(a), 6(a), and 7(a) describe the traffic jam of OVM. 


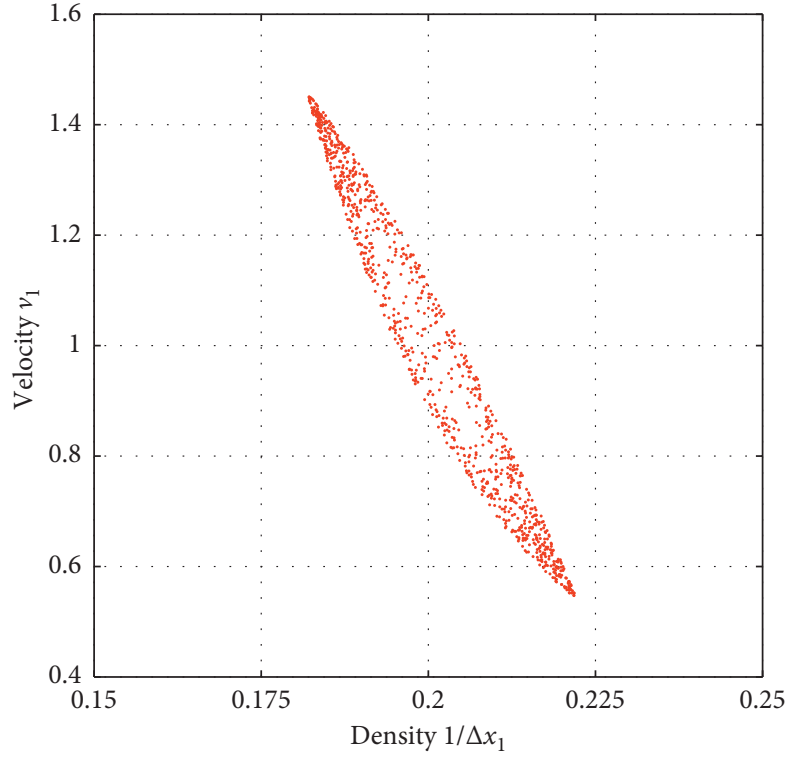

(a)

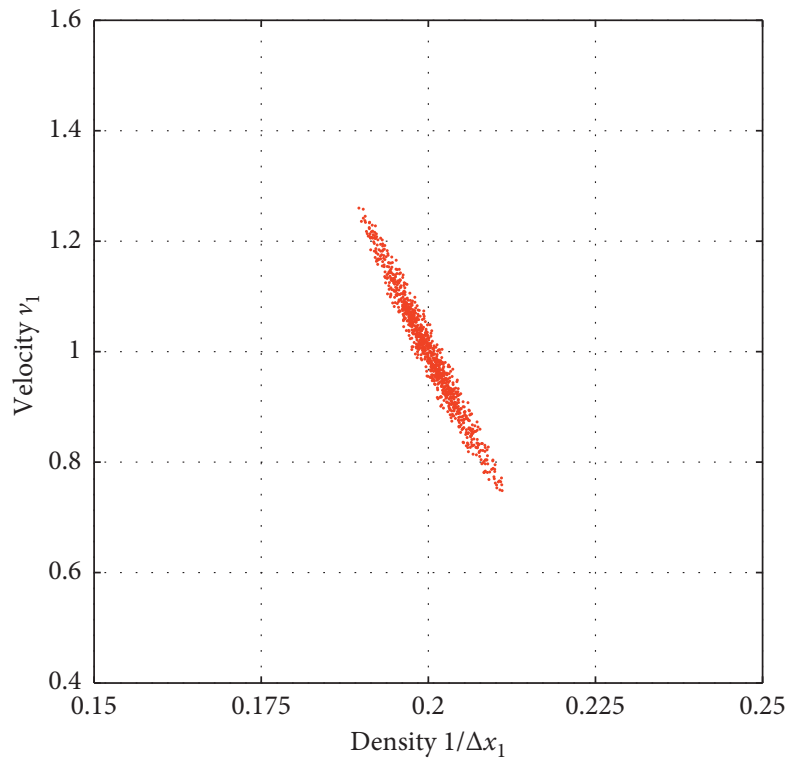

(c)

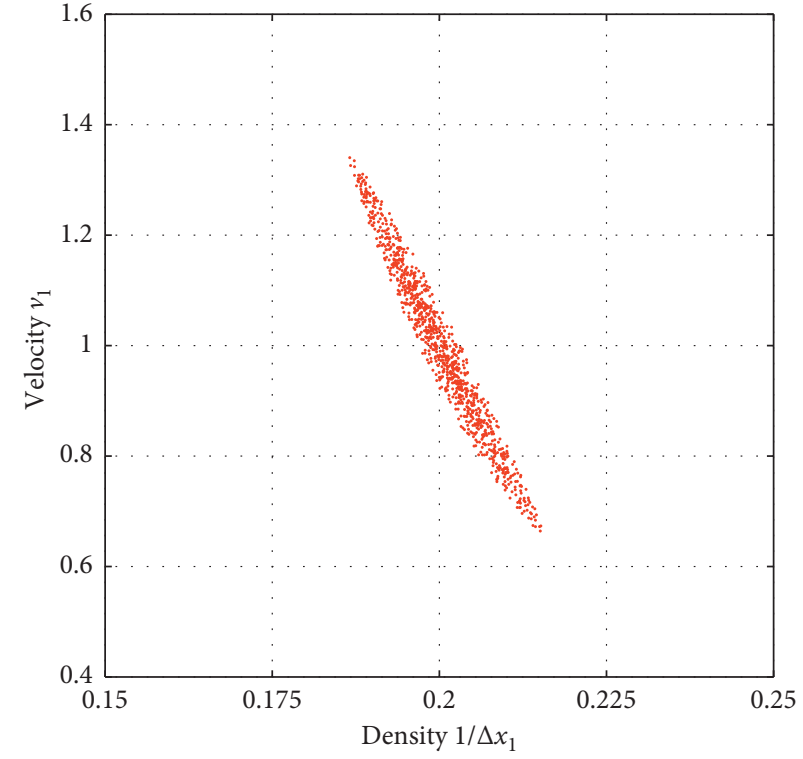

(b)

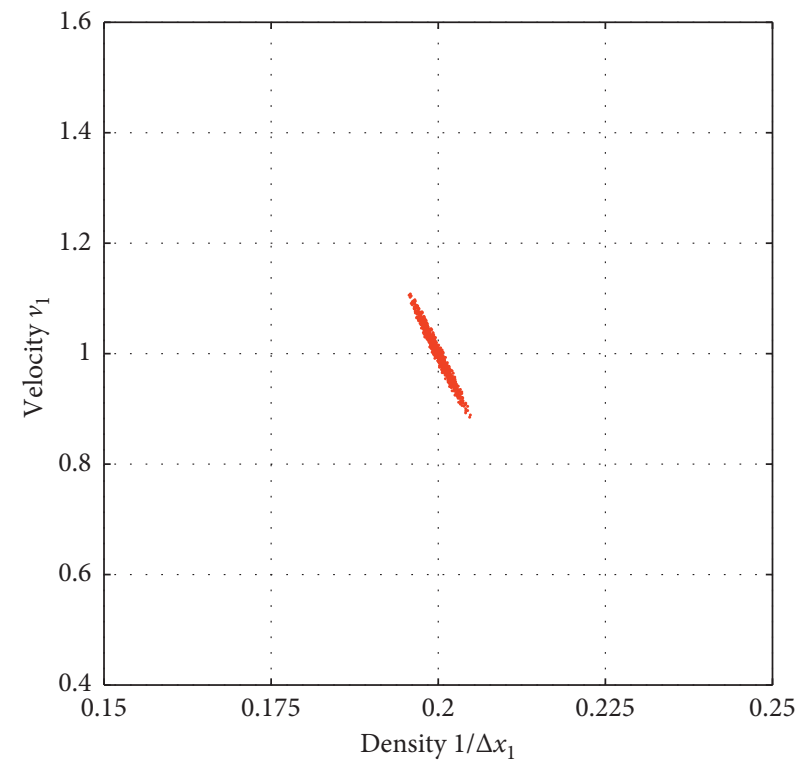

(d)

Figure 16: The hysteresis loop for different $\tau$ under $\lambda_{1}=0.01, \lambda_{2}=0.05$, and $p=0$. (a) $\tau=0$, (b) $\tau=0.75$, (c) $\tau=1$, and (d) $\tau=1.5$.

With increasing the value of $\lambda_{1}$, namely, the driver's desire for smooth driving is incorporated, we can observe that traffic jam is mitigated.

Then, in Figures $8-10, \lambda_{1}=0.01, p=0$, and $\tau=1$ are fixed, and $\lambda_{2}$ is a variable. In this case, the driver's desire for smooth driving and the self-stabilizing control effect are considered simultaneously. Three figures show the headway profiles, the velocity profiles, and the hysteresis loop, respectively. One can note that when increasing the value of $\lambda_{2}$ from 0 to 0.1 , the amplitude of traffic jam wave decreases, vehicles' velocity tends to be unified, and the hysteresis loop reduces to be a red dot. That is to say, the two factors considered can effectively diminish the traffic jam.
For investigating the effect of velocity uncertainty $p$ on traffic flow, fixed parameters are $\lambda_{1}=0.01, \lambda_{2}=0.05$, and $\tau=1$. Figures 11-13 represent the headway profiles, the velocity profiles, and the hysteresis loop, respectively. When $p<0$, the historical velocity perceived by driver is smaller than the real velocity, the driver overestimates the traffic condition and makes an aggressive response to increase the acceleration of vehicle, and the traffic accident may appear, so the traffic flow will be more unstable, while when $p>0$, the opposite is true, whereas the time delay will show up. Therefore, from Figures 11-13, what we can see is that the amplitude and the hysteresis loop of traffic congestion decrease by increasing the value of $p$ 
from negative to positive; however, the improvement of traffic congestion is at the price of unnecessary time delay, which conforms with the analysis above.

Finally, for analyzing the influence of time gap $\tau$, the coefficients of $\lambda_{1}, \lambda_{2}$, and $p$ are fixed to be $\lambda_{1}=0.01, \lambda_{2}=0.05$, and $p=0$. From Figures $14-16$, one can note that when increasing the value of $\tau$, the headway and the velocity of traffic flow in the first two figures tend to be stable; the hysteresis loop in the last figure shrinks gradually. Namely, the stability of traffic flow becomes higher.

\section{Conclusions}

This paper constructs an enhanced car-following model integrating the driver's desire for smooth driving and the self-stabilizing control effect. Both of the factors considered are based on the historical velocity data of current vehicle, while the current vehicle's historical velocity perceived by driver does not always conform with its real velocity. Therefore, the velocity uncertainty of current vehicle in the difference between actual velocity and historical velocity perceived is included in the model. For investigating the influences of these factors on traffic flow, the novel model is analyzed by linear and nonlinear stability analysis methods. At last, a series of simulation experiments are performed to verify the theoretical analytical results. The experimental results show that factors including the driver's desire for smooth driving and the self-stabilizing control can enhance the stability of traffic flow clearly. Then the larger historical time gap can improve the traffic congestion effectively. In addition, the value of velocity uncertainty can result in the degraded traffic efficiency $(p>0)$ and the traffic accident $(p<0)$.

\section{Data Availability}

The data used to support the findings of this study are available from the corresponding author upon request.

\section{Conflicts of Interest}

The authors declare that there are no conflicts of interest regarding the publication of this paper.

\section{Acknowledgments}

This work was supported by the Project of Humanities and Social Science of Education Ministry of China (Grant no. 20YJA630008), the Natural Science Foundation of Zhejiang Province, China (Grant no. LY20G010004), and the K.C. Wong Magna Fund in Ningbo University, China.

\section{References}

[1] Y. Sun, H. Ge, and R. Cheng, "An extended car-following model considering driver's memory and average speed of preceding vehicles with control strategy," Physica A: Statistical Mechanics and Its Applications, vol. 521, pp. 752-761, 2019.

[2] W.-X. Zhu and H. M. Zhang, "Analysis of mixed traffic flow with human-driving and autonomous cars based on car- following model," Physica A: Statistical Mechanics and Its Applications, vol. 496, pp. 274-285, 2018.

[3] T. Wang, R. J. Cheng, and H. X. Ge, "An extended two-lane lattice hydrodynamic model for traffic flow on curved road with passing," Physica A: Statistical Mechanics and Its Applications, vol. 533, Article ID 121915, 2019.

[4] T. Wang, R. J. Cheng, and H. X. Ge, "Analysis of a novel twolane lattice hydrodynamic model considering the empirical lane changing rate and the self-stabilization effect," IEEE Access, vol. 7, pp. 174725-174733, 2019.

[5] J. F. Wang, F. X. Sun, R. J. Cheng, and H. X. Ge, “An extended car-following model considering the self-stabilizing driving behavior of headway," Physica A: Statistical Mechanics and Its Applications, vol. 507, pp. 347-357, 2018.

[6] Z. Wen-Xing and Z. Li-Dong, "A new car-following model for autonomous vehicles flow with mean expected velocity field," Physica A: Statistical Mechanics and Its Applications, vol. 492, pp. 2154-2165, 2018.

[7] H. Ou and T.-Q. Tang, "An extended two-lane car-following model accounting for inter-vehicle communication," Physica A: Statistical Mechanics and Its Applications, vol. 495, pp. 260-268, 2018.

[8] T.-Q. Tang, X.-F. Luo, J. Zhang, and L. Chen, "Modeling electric bicycle's lane-changing and retrograde behaviors," Physica A: Statistical Mechanics and Its Applications, vol. 490, pp. 1377-1386, 2018.

[9] Y. Q. Sun, H. X. Ge, and R. J. Cheng, "An extended carfollowing model under V2V communication environment and its delayed-feedback control," Physica A: Statistical Mechanics and Its Applications, vol. 508, pp. 349-358, 2018.

[10] C. Chen, R. J. Cheng, and H. X. Ge, "An extended car-following model considering driver's sensory memory and the backward looking effect," Physica A: Statistical Mechanics and Its Applications, vol. 525, pp. 278-289, 2019.

[11] Q. Xin, N. Y. Yang, R. Fu, S. W. Yu, and Z. K. Shi, "Impacts analysis of car-following models considering variable vehicular gap policies," Physica A: Statistical Mechanics and Its Applications, vol. 501, pp. 338-355, 2018.

[12] T.-Q. Tang, Y.-X. Rui, J. Zhang, and H.-Y. Shang, "A cellular automation model accounting for bicycle's group behavior," Physica A: Statistical Mechanics and Its Applications, vol. 492, pp. 1782-1797, 2018.

[13] R. J. Cheng and Y. N. Wang, "An extended lattice hydrodynamic model considering the delayed feedback control on a curved road," Physica A: Statistical Mechanics and Its Applications, vol. 513, pp. 510-517, 2019.

[14] Q. Y. Wang and H. X. Ge, "An improved lattice hydrodynamic model accounting for the effect of "backward looking" and flow integral," Physica A: Statistical Mechanics and Its Applications, vol. 513, pp. 438-446, 2019.

[15] C. T. Jiang, R. J. Cheng, and H. X. Ge, "An improved lattice hydrodynamic model considering the "backward looking" effect and the traffic interruption probability," Nonlinear Dynamics, vol. 91, no. 2, pp. 777-784, 2018.

[16] X. Wu, X. M. Zhao, H. S. Song, Q. Xin, and S. W. Yu, "Effects of the prevision relative velocity on traffic dynamics in the ACC strategy," Physica A: Statistical Mechanics and Its Applications, vol. 515, pp. 192-198, 2019.

[17] Y. Y. Chang, Z. T. He, and R. J. Cheng, "An extended lattice hydrodynamic model considering the driver's sensory memory and delayed-feedback control," Physica A: Statistical Mechanics and Its Applications, vol. 514, pp. 522-532, 2019.

[18] Q. Y. Wang, R. J. Cheng, and H. X. Ge, "A new lattice hydrodynamic model accounting for the traffic interruption 
probability on a gradient highway," Physics Letters A, vol. 383, no. 16, pp. 1879-1887, 2019.

[19] J. F. Wang, F. X. Sun, and H. X. Ge, "An improved lattice hydrodynamic model considering the driver's desire of driving smoothly," Physica A: Statistical Mechanics and Its Applications, vol. 515, pp. 119-129, 2019.

[20] C. T. Jiang, R. J. Cheng, and H. X. Ge, "Effects of speed deviation and density difference in traffic lattice hydrodynamic model with interruption," Physica A: Statistical Mechanics and Its Applications, vol. 506, pp. 900-908, 2018.

[21] S. D. Qin, Z. T. He, and R. J. Cheng, "An extended lattice hydrodynamic model based on control theory considering the memory effect of flux difference," Physica A: Statistical Mechanics and Its Applications, vol. 509, pp. 809-816, 2018.

[22] C. T. Jiang, R. J. Cheng, and H. X. Ge, "Mean-field flow difference model with consideration of on-ramp and offramp," Physica A: Statistical Mechanics and Its Applications, vol. 513, pp. 465-476, 2019.

[23] Z. Z. Liu, R. J. Cheng, and H. X. Ge, "Research on preceding vehicle's taillight effect and energy consumption in an extended macro traffic model," Physica A: Statistical Mechanics and Its Applications, vol. 525, pp. 304-314, 2019.

[24] R. J. Cheng, H. X. Ge, and J. F. Wang, "KdV-Burgers equation in a new continuum model based on full velocity difference model considering anticipation effect," Physica A: Statistical Mechanics and Its Applications, vol. 481, pp. 52-59, 2017.

[25] R. J. Cheng, H. X. Ge, and J. F. Wang, "An improved continuum model for traffic flow considering driver's memory during a period of time and numerical tests," Physics Letters A, vol. 381, no. 34, pp. 2792-2800, 2017.

[26] Q. T. Zhai, H. X. Ge, and R. J. Cheng, "An extended continuum model considering optimal velocity change with memory and numerical tests," Physica A: Statistical Mechanics and Its Applications, vol. 490, pp. 774-785, 2018.

[27] R. J. Cheng, H. X. Ge, and J. F. Wang, "An extended macro traffic flow model accounting for multiple optimal velocity functions with different probabilities," Physics Letters A, vol. 381, no. 32, pp. 2608-2620, 2017.

[28] T. Q. Tang, H. J. Huang, and G. Xu, "A new macro model with consideration of the traffic interruption probability," Physica A: Statistical Mechanics and Its Applications, vol. 387, no. 27, pp. 6845-6856, 2008.

[29] R. J. Cheng, H. X. Ge, and J. F. Wang, "The nonlinear analysis for a new continuum model considering anticipation and traffic jerk effect," Applied Mathematics and Computation, vol. 332, pp. 493-505, 2018.

[30] Z. H. Wang, R. J. Cheng, and H. X. Ge, "Nonlinear analysis of an improved continuum model considering mean-field velocity difference," Physics Letters A, vol. 383, no. 7, pp. 622-629, 2019.

[31] R. J. Cheng, H. X. Ge, and J. F. Wang, "An extended continuum model accounting for the driver's timid and aggressive attributions," Physics Letters A, vol. 381, no. 15, pp. 1302-1312, 2017.

[32] Z. H. Wang, H. X. Ge, and R. J. Cheng, "Nonlinear analysis for a modified continuum model considering driver's memory and backward looking effect," Physica A: Statistical Mechanics and Its Applications, vol. 508, pp. 18-27, 2018.

[33] W.-X. Zhu, D. Jun, and L.-D. Zhang, "A compound compensation method for car-following model," Communications in Nonlinear Science and Numerical Simulation, vol. 39, pp. 427-441, 2016.

[34] T. Q. Tang, J. Zhang, and K. Liu, “A speed guidance model accounting for the driver's bounded rationality at a signalized intersection," Physica A: Statistical Mechanics and Its Applications, vol. 473, pp. 45-52, 2017.

[35] W.-X. Zhu and L.-D. Zhang, "Analysis of car-following model with cascade compensation strategy," Physica A: Statistical Mechanics and Its Applications, vol. 449, pp. 265-274, 2016.

[36] H. Kuang, Z.-P. Xu, X.-L. Li, and S.-M. Lo, "An extended carfollowing model accounting for the average headway effect in intelligent transportation system," Physica A: Statistical Mechanics and Its Applications, vol. 471, pp. 778-787, 2017.

[37] L. A. Pipes, "An operational analysis of traffic dynamics," Journal of Applied Physics, vol. 24, no. 3, pp. 274-281, 1953.

[38] G. F. Newell, "Nonlinear effects in the dynamics of car following," Operations Research, vol. 9, no. 2, pp. 209-229, 1961.

[39] K. Bando, M. Hasebe, A. Nakayama, A. Shibata, and Y. Sugiyama, "Dynamical model of traffic congestion and numerical simulation," Physical Review E, vol. 51, no. 2, pp. 1035-1042, 1995.

[40] D. Helbing and B. Tilch, "Generalized force model of traffic dynamics," Physical Review E, vol. 58, no. 1, pp. 133-138, 1998.

[41] R. Jiang, Q. S. Wu, and Z. J. Zhu, "Full velocity difference model for a car-following theory," Physical Review E, vol. 64, no. 1, Article ID 017101, 2001.

[42] J. F. Wang, F. X. Sun, and H. X. Ge, "Effect of the driver's desire for smooth driving on the car-following model," Physica A: Statistical Mechanics and Its Applications, vol. 512, pp. 96-108, 2018.

[43] Z. P. Li, Q. Q. Qin, W. Z. Li, S. Z. Xu, Y. Q. Qian, and J. Sun, "Stabilization analysis and modified KdV equation of a carfollowing model with consideration of self-stabilizing control in historical traffic data," Nonlinear Dynamics, vol. 91, no. 2, pp. 1113-1125, 2018.

[44] G. Zhang, D.-H. Sun, M. Zhao, X.-Y. Liao, W.-N. Liu, and T. Zhou, "An extended car-following model accounting for cooperation driving system with velocity uncertainty," Physica A: Statistical Mechanics and Its Applications, vol. 505, pp. 1008-1017, 2018.

[45] T. Nagatani, "Modified KdV equation for jamming transition in the continuum models of traffic," Physica A: Statistical Mechanics and Its Applications, vol. 261, no. 3-4, pp. 599-607, 1998. 